\title{
Dynamic Characteristics Analysis and Test of Dual-Driving Feed System Driven by Center of Gravity
}

\author{
Hong Lu, ${ }^{1,2}$ Wei Fan, ${ }^{1}$ Xinbao Zhang $\left(\mathbb{D},{ }^{3}\right.$ Yongquan Zhang, \\ Shaojun Wang, ${ }^{4}$ and Meng Duan ${ }^{1}$ \\ ${ }^{1}$ School of Mechanical and Electronic Engineering, Wuhan University of Technology, Hubei, China \\ ${ }^{2}$ Key Laboratory of Hubei Province for Digital Manufacture, Hubei, China \\ ${ }^{3}$ School of Mechanical Science and Engineering, Huazhong University of Science and Technology, Hubei, China \\ ${ }^{4}$ School of Industrial and Engineering Technology, Southeast Missouri State University, Cape Girardeau, MO, USA
}

Correspondence should be addressed to Xinbao Zhang; zhangxinbao1@hust.edu.cn

Received 2 August 2017; Revised 10 December 2017; Accepted 31 December 2017; Published 31 January 2018

Academic Editor: Xinkai Chen

Copyright (C) 2018 Hong Lu et al. This is an open access article distributed under the Creative Commons Attribution License, which permits unrestricted use, distribution, and reproduction in any medium, provided the original work is properly cited.

\begin{abstract}
Dual-driving feed system (DDFS) driven by center of gravity (DCG) has been widely used in advanced manufacturing machine for its high rigidity and precision. However, the DCG technology requires that the joint force coincides with the center of gravity of the sliding stage. The dual-driving synchronization and tracking performance will be affected by the change of center of gravity of the sliding stage. Therefore, this paper proposes dynamic characteristics modeling, identification, and control scheme for DDFS driven by center of gravity (DCG). Firstly, a redundancy dynamic model including rotation and pitch vibration caused by the change of the position of center of gravity is presented for DDFS DCG based on the Lagrange method. The model parameters are identified by system identification experiment, and the predictive natural frequencies and vibration modes by the proposed dynamic model are compared by modal experiment. Moreover, the dynamic model-based cross-coupled sliding mode control (CCSMC) is proposed for DDFS DCG. Then, the proposed dynamic model-based CCSMC has been compared with normal cross-coupled sliding mode control (NCCSMC). Both the simulation and experimental results show that the proposed dynamic characteristics analysis and test scheme of DDFS DCG are validated effectively by comparisons.
\end{abstract}

\section{Introduction}

Traditional drive mechanism used single servo motor and ball screw in gantry-moving machine. However, in this single drive structure, the deformation and vibration of screw are inevitably caused by the deviation between the drive force and center of gravity. Furthermore, the load capacity is restricted owing to the limit of the power and torque of single servo motor. The DDFS is used on high-end industrial applications such as surface chip mounting, precision metrology, and high-speed and heavy load manufacturing [1]. Since the joint force is provided by twin motors and screws, the system rigidity would be significantly increased, and double overall thrust would afford to drive the stage.

Moreover, based on driving by center of gravity (DCG), DDFS can improve the system dynamics with high acceleration and low vibration. The DCG technology is innovatively investigated by Mori Seiki. This twin ball screws structure could ensure the direction of drive forces close to the center of stage gravity, so that the vibration of machine tools could be decreased dramatically [2].

Numerous researches have conducted ball screw system and DDFS in CNC machine tools [3-6]. Most of them focused on the synchronous control algorithm based on master-slave or cross-coupled control structure and simplified the mechanical system as transmission ratio for simply research [7, 8]. The synchronous control strategies have certain effects to improve the synchronization performance for common DDFS. However, the mechanical characteristics diversity and coupling factor of DDFS are ignored in these researches. In fact, the performance of DDFS suffered from the diversity of mechanism characteristics, deflection of joint thrust, and mechanical coupling [9-12]. To improve 
the synchronous performance, some scholars established the mechanical coupling models by identification technique [13$15]$, yet the models are established as first-order inertia links, and the parameters are identified without actual physical meaning [16].

Furthermore, as to the dynamic modeling based on DCG technology, not only the differences of servo and mechanical characteristics between the two axis but also the change of the center of gravity of the sliding stage should be considered. Nevertheless, most researches seldom consider the effect of position of center of gravity. Most of the researches assume that the DCG feed drive is implemented on a horizontal plane, and the position of center of gravity always remains invariant. Actually, if the sliding stage was mounted with different loads, or the position of the sliding stage along the direction $Y$, perpendicular to dual-driving feed direction, was changed, the synchronization of the DDFS will be inevitably affected. Since the height of center of gravity does not equal zero, both torsion vibration and pitch mode should be taken into consideration in dynamic research of dual-driving system.

In addition, the synchronization and tracking accuracy of DDFS are determined by the bandwidth of servo control loop $[17,18]$. The bandwidth of the DDFS is still limited by the structural resonances arising from the mechanical structure [19]. Especially in high acceleration motion, the bandwidth is limited by a specific vibration mode in which the dual-driving stage oscillates as the double ball screws undergo torsion and pitch vibrations. Some researches built the dynamic model by considering vibration characteristics of feed system in CNC machine tool [20-22]. These are helpful for us to analyze the dynamic characteristics of DDFS DCG.

As to the synchronous control, many scholars have developed the motion control strategy for DDFS from the perspective of tandem control and cross-coupled control [23]. In the former case, the tandem control is the most widely used method in industry and the DDFS is distinguished as master and slave axis. The output of the master motor is considered as the input of the slave motor. The slave motor has to follow the exact position of the master motor to maintain synchronization. However, the disturbance of the slave axis cannot affect the controller of the master axis, and the synchronization accuracy needs to be improved. In the second one, the DDFS is considered as two coupled axes, and the parameters variation is considered as disturbances. The cross-coupled controller between the dual axes can compensate the synchronous error. However, to avoid the large overshoot of dual axis following each other [24], the dynamic characteristics caused by parameters variation and coupling factor of DDFS need to be modeled and analyzed.

Hence, in order to help to analyze the natural frequency and dynamic characteristics of DDFS DCG and also benefit the synchronous control, the redundancy dynamic model including rotation and pitch errors for DDFS DCG is proposed in this paper. The contributions of this work can be listed as follows:

(i) The redundancy dynamic model including rotation and pitch errors of the DDFS DCG is developed. This model is different from the previous mathematical model which considering the center of gravity is in the geometric center. In this way, the influence because of the time-varying position change of center of gravity of the sliding stage is theoretically discussed and experimentally validated. Moreover, the variation parameters of dual axis are obtained by practical identification experiment.

(ii) The dynamic model-based CCSMC has been proposed for DDFS DCG. The maximum and average synchronous errors of the proposed control scheme are compared with NCCSMC. The comparison results show the effectiveness of the proposed model and control scheme.

(iii) This work focuses on the practical application of highprecision feed system in machine tools. The proposed model of used dual-driving stage is verified by natural frequency with the increasing of height of center of gravity; the value and changing trend of simulated natural frequency is tested by experimental results.

The structure is organized as follows. In Section 2, the redundancy dynamic model including rotation and pitch errors for DDFS DCG is developed, along with the DCG mechanism analysis. Section 3 concerns the validation and comparison of the proposed model by practical DDFS DCG. The dynamic model-based CCSMC is presented in Section 4. The experimental results show the effectiveness of the proposed dynamic analysis and test method. Conclusions are drawn in Section 5.

\section{Dynamic Modeling and Parameters Identification of DDFS DCG}

The DDFS DCG used in this paper is composed of twin AC200-v permanent magnet synchronous motor (PMSM) and ball screws; namely, on each feed direction, the table is driven by two motors and two screws joint together. A simplified sketch of the proposed typical gantry stage with DCG DDFS is illustrated in Figure 1. In the DDFS DCG, the one-axis movement is achieved by parallel twin motors and screws. Two ball screws aligned in parallel guide to move the table in $y$-axis, and this moving table serves as the base of the sliding stage which moves along $x$-axis.

The dual-driving stage is a high-speed, high-precision positioning system. It consists of a dual-driving stage that is moved by two parallel ball screws (axes $y_{1}$ and $y_{2}$ ). The stage is mounted on the geometry center so that the initial coordinate of center of gravity $\left(x_{0}, y_{0}, z_{0}\right)$ locates on the resultant forces. At this case, the DCG dual-driving can be achieved if the driving forces are equal. However, as can be seen in Figure 1, the feed accuracy of DDFS is suffered from the diversity of servo and mechanical characteristics, deflection of joint thrust, frictions, and so on. Moreover, the stage moved along $x$-axis, and different workpiece which mounted on the stage will increase the height of center of gravity. The dynamics of DDFS should be considered based on transmission coordinate $\left(x_{g}, y_{g}, z_{g}\right)$. The travel length 

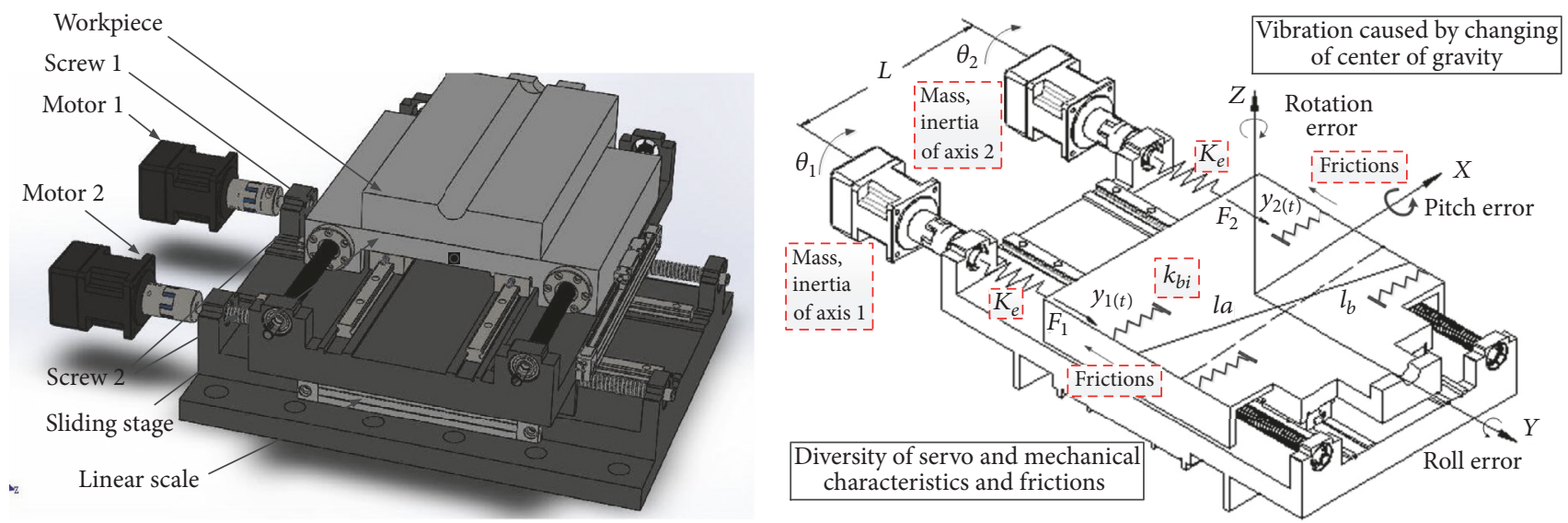

FIGURE 1: Basic structure and mechanism of DCG DDFS.

along $x$-axis can vary from the middle position to the lefthand (or right-hand) side. And the height of center of gravity is increased depending on the application and work required.

2.1. Redundancy Dynamic Model of DCG DDFS. The proposed redundancy dynamic model of DCG DDFS is developed in this section. Based on the dual-driving structure and mechanism shown in Figure 1, the lumped-parameter model is described in Figures 2 and 3. The lumped model is described based on the change of position of center of gravity. In this model, the new coordinate system considers not only the linear positions of the sliding stage, but also the rotation and pitch around center of gravity of the sliding stage.

As can be seen in Figures 2 and 3, the parameters are defined as follows:

$m_{y i}$ is the equivalent mass of each axis.

$m_{s}$ is the mass of the sliding stage.

$y_{i}$ is the actual linear displacement of each axis.

$F_{e i}$ is the equivalent force of each axis.

$X_{g}, Y_{g}$, and $Z_{g}$ are the displacements between $\left(x_{g}, y_{g}, z_{g}\right)$ and $\left(x_{0}, y_{0}, z_{0}\right)$ in $x$-axis, $Y$-axis, and $z$ axis, respectively.

$\theta_{g x}$ is the pitch angle around $x$-axis; $\theta_{g z}$ is the rotation angle around $z$-axis.

$k_{h z}$ is the support stiffness of slider; $k_{b x}$ is the later stiffness of slider.

$k_{e}$ is the equivalent axial stiffness of screw systems, which would be identified by actual feed system.

$l_{1}$ and $l_{2}$ are the displacement from force points of screws to center of mass in the sliding stage.

$L$ is the distance of dual screws.

$a$ is the axial distance of sliders.

The drive force can be obtained from equivalent axial stiffness:

$$
\begin{aligned}
& F_{1}=k_{e}\left(y_{1}-y_{s 1}\right), \\
& F_{2}=k_{e}\left(y_{2}-y_{s 2}\right),
\end{aligned}
$$

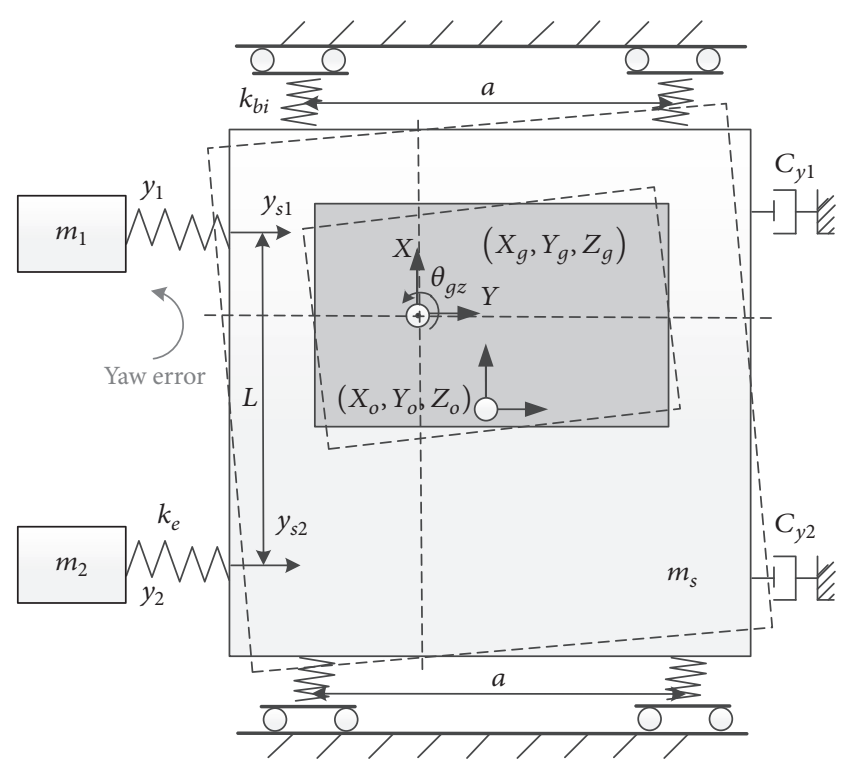

FIGURE 2: Lumped-parameter model considers rotation around $z$ axis.

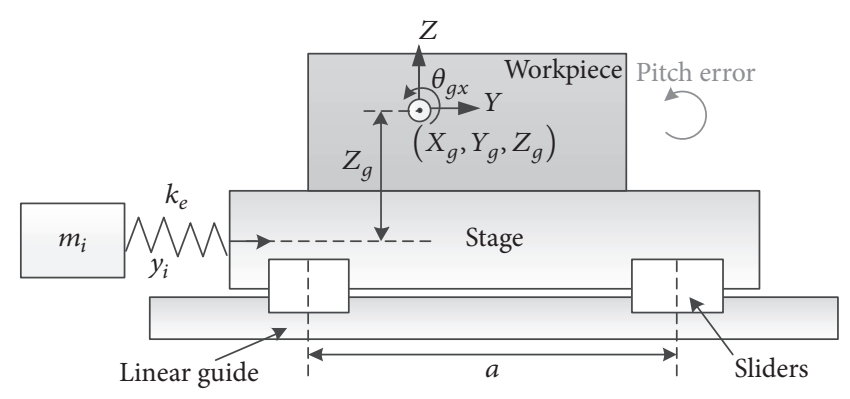

FIGURE 3: Lumped-parameter model considers pitch around $x$-axis.

where $y_{s 1}$ and $y_{s 2}$ are the equivalent linear displacements of double servo motors. 
The total drive force of table can be deduced as

$$
\begin{aligned}
F & =F_{e 1}+F_{e 2}, \\
F_{e 1} & =F_{1}-c_{y_{1}} \operatorname{sign}\left(\dot{y}_{1}\right), \\
F_{e 2} & =F_{2}-c_{y_{2}} \operatorname{sign}\left(\dot{y}_{2}\right) .
\end{aligned}
$$

$c_{y_{1}}$ and $c_{y_{2}}$ are forces corresponding to equivalent total friction of axis $y_{1}$ and $y_{2}$.

When the workpiece is not clamped on the sliding stage, the drive torque of DDFS DCG structure around center of gravity is zero, and the pitch error will not be produced. After clamping the workpiece, according to the geometric relationship, the driving torque can be converted from the driving forces, that is

$$
T_{g x}=\left(F_{e 1}+F_{e 2}\right) Z_{g} .
$$

The driving torque of DCG dual-driving structure is smaller than normal single driving structure for its shorter arm of force. However, once the workpiece is installed on the stage, the driving torque is generated for the height of center of gravity is not zero. The pitch errors are also generated and cannot be compensated by adjusting the driving forces of dual axis.

The lumped-parameter model of DCG dual-driving stage is shown in Figure 2. The fundamental coordinate is established based on the original center of gravity $O_{o}\left(x_{0}, y_{0}, z_{0}\right)$. $\mathrm{O}_{o}$ is center of gravity of dual-driving stage without clamping the workpiece, which is designed to be on the horizon plane of axes of double screws. However, when the workpiece is clamped and moved along $x$-axis, the translation coordinate system is built based on the actual center of gravity $\mathrm{O}_{g}\left(x_{g}, y_{g}, z_{g}\right)$. During the dual-driving process in $y$-axis, the stage is driven by dynamic joint forces. Thus, the rotation around $z$-axis and pitch vibration around $x$-axis will be induced and impact on the feed accuracy.

Since the yaw angle of cross-arm is limited to nearly $0.1 \mathrm{rad}$, the $\sin \theta_{g z}$ can be considered as $\theta_{g z}$, and $\cos \theta_{g z}$ is simplified to be 1 . Considering the torsion and pitch vibration, the displacements of dual axis are given by

$$
\begin{aligned}
\operatorname{Rot}\left(x, \theta_{g x}\right) & =\left[\begin{array}{cccc}
1 & 0 & 0 & 0 \\
0 & \cos \theta_{g x} & \sin \theta_{g x} & 0 \\
0 & -\sin \theta_{g x} & \cos \theta_{g x} & 0 \\
0 & 0 & 0 & 1
\end{array}\right], \\
\operatorname{Rot}\left(z, \theta_{g z}\right) & =\left[\begin{array}{cccc}
\cos \theta_{g z} & \sin \theta_{g z} & 0 & 0 \\
-\sin \theta_{g z} & \cos \theta_{g z} & 0 & 0 \\
0 & 0 & 1 & 0 \\
0 & 0 & 0 & 1
\end{array}\right] .
\end{aligned}
$$

Then, the displacements of each axis can be expressed as

$$
\begin{aligned}
& y_{1}=y-\left(\frac{L}{2}-X_{g}\right) \theta_{g z}+Z_{g} \theta_{g x}=y-l_{1} \theta_{g z}+Z_{g} \theta_{g x}, \\
& y_{2}=y+\left(\frac{L}{2}+X_{g}\right) \theta_{g z}+Z_{g} \theta_{g x}=y+l_{2} \theta_{g z}+Z_{g} \theta_{g x},
\end{aligned}
$$

where $y_{1}$ and $y_{2}$ are the displacements of the nuts of each axis and $y$ is the displacement of the stage in $Y$ direction. Since the actuator is mounted on the stage, $y$ also represented the actual displacement of actuator. From (5) we can see that $y$ is affected by the position of the center of gravity, the pitch errors $\theta_{g x}$, and yaw errors $\theta_{g z}$, which means that the research of position of center of gravity is necessary to improve the feed accuracy. The yaw errors $\theta_{g z}$ are reflected in the synchronous errors. In order to improve the tracking accuracy, the pitch errors $\theta_{g x}$ caused by the increase of center of gravity are required to be noticed and compensated.

The microrotation angle can be expressed as

$$
\theta_{g z}=\frac{\left(y_{2}-y_{1}\right)}{L} \text {. }
$$

Then the motion equations of the DCG dual-driving stage will be derived using Lagrange-Euler formalism. Based on the conservation of energy, Lagrange-Euler equation can be used in the dynamics modeling and analysis of multidegree of freedom system. Through the eigenvalue decomposition, the natural frequencies and vibration modes of DDFS DCG can be obtained. To this purpose, the kinetic energy of the DCG dual-driving system can be expressed as

$$
\begin{aligned}
T= & \frac{1}{2}\left(m_{y 1} \dot{y}_{1}^{2}+m_{y 2} \dot{y}_{2}^{2}\right)+\frac{1}{2} m_{s} \dot{y}^{2}+\frac{1}{2} J_{z} \dot{\theta}_{g z}^{2} \\
& +\frac{1}{2} J_{x} \dot{\theta}_{g x}^{2} .
\end{aligned}
$$

Thus, two sets of geometric relationships in $x$-axis and $z$-axis can be used to specify the displacements of slide blocks. $x_{b i}$ are the linear displacements of slide blocks in the direction of $x$-axis. The relationship between $x_{b i}$ and torsion angle $\theta_{g z}$ is given by

$$
\begin{aligned}
& x_{b 1,3}=x_{g} \pm\left(\frac{a}{2}-Y_{g}\right) \theta_{g z}, \\
& x_{b 2,4}=x_{g} \mp\left(\frac{a}{2}+Y_{g}\right) \theta_{g z} .
\end{aligned}
$$

And the relation between displacements of slide blocks in $z$-axis and pitch angle $\theta_{g x}$ is given by

$$
\begin{aligned}
& z_{b 1,3}=\left(\frac{a}{2}-Y_{g}\right) \theta_{g x}, \\
& z_{b 2,4}=\left(\frac{a}{2}+Y_{g}\right) \theta_{g x} .
\end{aligned}
$$

The dual-driving stage is designed to be symmetrical, and the center of gravity is designed to be located on the geometric center as mentioned. Compared to the deflections of center of gravity in $x$-axis and $z$-axis, the deflection in $y$-axis is negligible when clamping workpiece of different weights.

The potential elastic energy can be obtained

$$
\begin{aligned}
U= & \frac{1}{2} k_{e 1}\left(y_{s 1}-y_{1}\right)^{2}+\frac{1}{2} k_{e 2}\left(y_{s 2}-y_{2}\right)^{2}+\frac{1}{2} k_{b x} \sum_{i=1}^{4} x_{i}^{2} \\
& +\frac{1}{2} k_{b z} \sum_{i=1}^{4} z_{i}^{2} .
\end{aligned}
$$


According to Lagrange method, the system dynamics can be defined as

$$
\frac{d}{d t}\left(\frac{\partial L}{\partial \dot{q}_{j}}\right)-\frac{\partial L}{\partial q_{j}}=Q_{j},
$$

where

$$
\begin{aligned}
& q_{1}=y_{1}, \\
& q_{2}=y_{2}, \\
& q_{3}=x, \\
& q_{4}=\theta_{g z}, \\
& q_{5}=\theta_{g x}, \\
& L=T-U .
\end{aligned}
$$

$$
\begin{aligned}
& M=\left(\begin{array}{ccccc}
m_{y 1} & 0 & 0 & 0 & 0 \\
0 & m_{y 2} & 0 & 0 & 0 \\
0 & 0 & m_{s} & 0 & 0 \\
0 & 0 & 0 & J_{g z} & 0 \\
0 & 0 & 0 & 0 & J_{g x}
\end{array}\right) \\
& K=\left(\begin{array}{ccccc}
k_{e} & 0 & -k_{e} & l_{1} k_{e} & -Z_{g} k_{e} \\
0 & k_{e} & -k_{e} & -l_{2} k_{e} & -Z_{g} k_{e} \\
-k_{e} & -k_{e} & 2 k_{e} & \left(l_{2}-l_{1}\right) k_{e} & 2 Z_{g} k_{e} \\
l_{1} k_{e} & -l_{2} k_{e} & \left(l_{2}-l_{1}\right) k_{e} & \left(l_{1}^{2}+l_{2}^{2}\right) k_{e}+a^{2} k_{b x} & \left(l_{1}-l_{2}\right) Z_{g} k_{e} \\
-Z_{g} k_{e} & -Z_{g} k_{e} & 2 Z_{g} k_{e} & \left(l_{2}-l_{1}\right) Z_{g} k_{e} & 2 Z_{g}^{2} k_{e}+a^{2} k_{b z}
\end{array}\right), \\
& F=\left(\begin{array}{c}
F_{e 1} \\
F_{e 2} \\
0 \\
T_{g z} \\
T_{g x}
\end{array}\right)\left\{\begin{array}{l}
F_{e 1}=k_{e 1}\left(y_{1}-y_{s 1}\right)-c_{y_{1}} \operatorname{sign}\left(\dot{y}_{1}\right) \\
F_{e 2}=k_{e 2}\left(y_{2}-y_{s 2}\right)-c_{y_{2}} \operatorname{sign}\left(\dot{y}_{2}\right) \\
T_{g z}=F_{e 2} l_{2}-F_{e 1} l_{1} \\
T_{g x}=\left(F_{e 1}+F_{e 2}\right) Z_{g} .
\end{array}\right.
\end{aligned}
$$

The stiffness matrix $K$ points out to the mechanical coupling between the dual-driving axes $y_{1}$ and $y_{2}$ in the multidegree of freedom, which is affected by equivalent stiffness $k_{e}, k_{b x}, k_{b z}$, and different positions of the center of gravity $\left(l_{2}-l_{1}\right), Z_{g}$. The simplified inertia and damping matrices show the nonuniform load and friction distribution. The first two lines of $F$ are the force vector. Assume that $l_{1}=l_{2}, m_{y 1}=m_{y 2}, c_{y 1}-c_{y 2}=0$, and $y_{1}-y_{s 1}=y_{2}-y_{s 2}$, which means the mass, inertia, and friction of dual axes are equal and the workpiece is located on the middle of the stage. In this case, dual-driving synchronous feed can be ensured by $F_{e 1}=F_{e 2}$, which represent that the equivalent forces are equal and the joint force coincides with the center of gravity in $X$ $Y$ plane. However, due to the different mechanical and servo characteristics of dual axis, and the center of gravity moving along $x$ - and $z$-axis, the perfect synchronous dual-driving is hard to be achieved.

Moreover, the last two lines of the force vector $F$ are torques around $z$-axis and $x$-axis, respectively. Traditionally, the dynamic model of DDFS is established based on two degrees of freedom, the linear displacement in $y$-axis and rotation angle around $z$-axis; the pitch errors caused by increase of position of center of gravity are totally ignored $[5,12] . T_{g x}$ is the torque around $x$-axis associated with the deviation of center of gravity in height. Only if the center of gravity is located on the $X, Y$ plane, which means $Z_{g}=0$, the torque around $x$-axis approaches zero. However, when the workpiece with different weight is located, it is impossible to 
ensure $Z_{g}=0$. The joint force will not go through the center of gravity and pitch error would be generated. To achieve satisfactory DCG feed accuracy, the dynamic of pitch and torsion vibrations should be measured and analyzed in the dual-driving process.
The determinant of the Lagrange equation can be obtained as

$$
\begin{aligned}
& D=\left(\begin{array}{ccccc}
A_{11} & 0 & -k_{e} & l_{1} k_{e} & -Z_{g} k_{e} \\
0 & A_{22} & -k_{e} & -l_{2} k_{e} & -Z_{g} k_{e} \\
-k_{e} & -k_{e} & A_{33} & \left(l_{2}-l_{1}\right) k_{e} & 2 Z_{g} k_{e} \\
l_{1} k_{e} & -l_{2} k_{e} & \left(l_{2}-l_{1}\right) k_{e} & A_{44} & \left(l_{1}-l_{2}\right) Z_{g} k_{e} \\
-Z_{g} k_{e} & -Z_{g} k_{e} & 2 Z_{g} k_{e} & \left(l_{2}-l_{1}\right) Z_{g} k_{e} & A_{55}
\end{array}\right) \\
& A_{11}=-m_{y 1}^{2} w^{2}+k_{e} A_{22}=-m_{y 2}^{2} w^{2}+k_{e}, \\
& A_{33}=-2 m_{s}^{2} w^{2}+k_{e}, \\
& A_{44}=-J_{g z} w^{2}+\left[\left(l_{1}^{2}+l_{2}^{2}\right) k_{e}+a^{2} k_{b x}\right] \\
& A_{55}=-J_{g x} w^{2}+\left[2 Z_{g}^{2} k_{e}+a^{2} k_{b z}\right] .
\end{aligned}
$$

The determinant (17) expresses the axial, pitch, and torsion resonant modes. Solve the determinant $D=0 ; w_{i}(i=$ $1 \cdots 5$ ) represent the natural frequencies.

2.2. Model Parameters Identification. As to (17) and (18), the dynamic parameters can be divided into three parts: inertia parameters (the inertia matrix $M$ ), jointed parameters (the stiffness matrix $K$ and damping matrix $C$ ), and structure parameters (the constant parameters such as $a, L$ ). For the determined dual-driving DCG feed system, the structure parameters are assumed to be constant during the machining process. Hence, only the inertia and jointed parameters are analyzed and identified in this section.

2.2.1. Test Platform Setup. As shown in Figures 4 and 5, DDFS and National Instruments (NI) test platform are set up for system parameters identification. The DDFS is constructed by Beckhoff servo system and controller, which will also be employed in the feed verification experiment later. The sweep sine signal is generated as identification input by the dynamic signal analyzer (DSA) from NI. The frequency response data is also calculated and analyzed in DSA. A data acquisition (DAQ) card inserted in a $P C$ is used to receive the analog signal. All the AD/DA conversion, system memory allocation, and accessing module are executed by controller of NI. The detection data is analyzed by LabVIEW frequency response analyzer code.

The sweep sine input signal applied to the drive is generated by DSA module. And the same signal is also linked to the Beckhoff controller or amplifier. The velocity and acceleration limits of double motors are $1.5 \mathrm{~m} / \mathrm{s}$ and $5 \mathrm{~m} / \mathrm{s}^{2}$.
The desired motion trajectories of dual-driving axis are given exactly the same to avoid desynchronization.

2.2.2. Parameters Identification. As a major component of a dual-driving NC machine tool, the stiffness of the ball screws and linear guides are crucial to affect the positioning accuracy directly. Normally, the axial stiffness $k_{e}$ of screw system is estimated as the related components in series and parallel. The screw, nut, and support bearing influence the stiffness most. The dynamic model is shown in Figure 6.

The integral axial stiffness of the screw transmission system is made up of the stiffness of screw, nut joint, and support bearing joints series-parallel connections. The equivalent axial stiffness can be given by

$$
\begin{aligned}
& k_{e} \\
& =\frac{\left(\left(k_{s 1\left(y_{b}\right)}+k_{b 1}\right) / k_{s 1\left(y_{b}\right)} k_{b 1}+\left(k_{s 2\left(y_{b}\right)}+k_{b 2}\right) / k_{s 2\left(y_{b}\right)} k_{b 2}\right)+k_{\text {nut }}}{\left(\left(k_{s 1\left(y_{b}\right)}+k_{b 1}\right) / k_{s 1\left(y_{b}\right)} k_{b 1}+\left(k_{s 2\left(y_{b}\right)}+k_{b 2}\right) / k_{s 2\left(y_{b}\right)} k_{b 2}\right) k_{\text {nut }}} .
\end{aligned}
$$

The equivalent axial stiffness can be obtained from the tension and torsion stiffness when the structure is determined, and this axial stiffness function is only affected by the located position of nut.

Different frictional characteristics will generate the unbalanced forces in double axes. So the frictions model should be built accurately. The nonlinearity friction model is described as [11]

$$
c_{y(v)}=F_{C}+\left(F_{S}-F_{C}\right) e^{-\left|v / v_{\sigma}\right|^{\delta_{\sigma}}}+F_{v} v \quad(v \neq 0),
$$

where $v_{\sigma}$ is the Stribeck velocity, $F_{C}$ is Coulomb friction, and $F_{S}$ is the maximum static friction. According to Newton's 


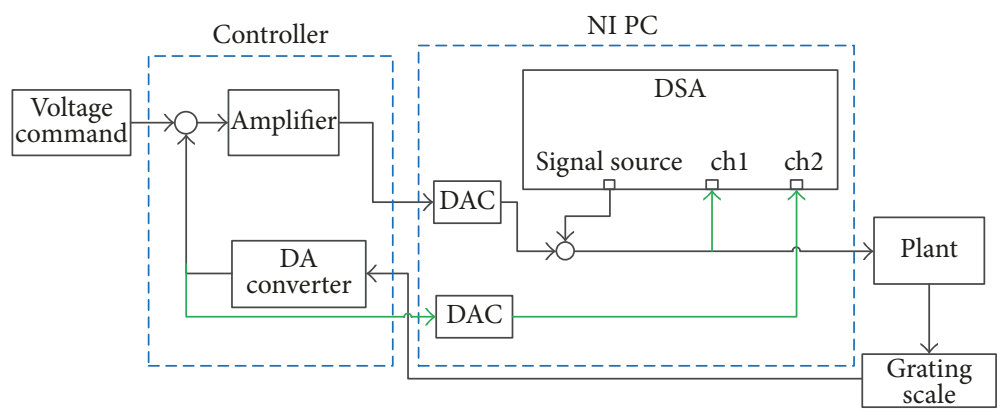

FIGURE 4: Frequency response experiment diagram.

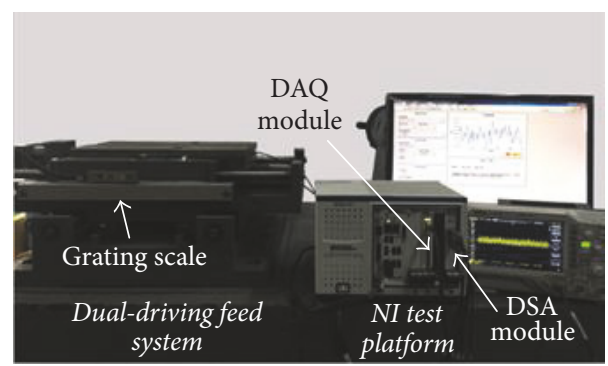

Figure 5: Experiment setup of identification.

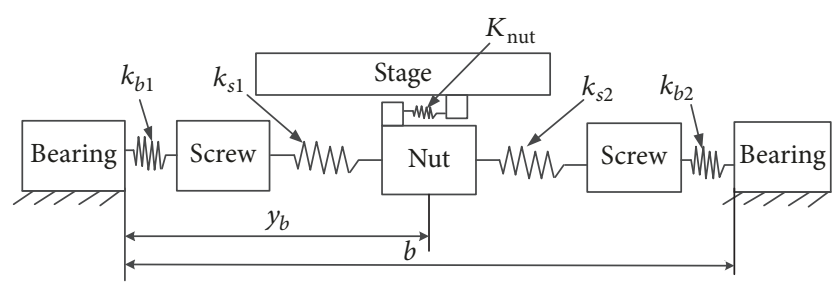

FIGURE 6: Simplified axial stiffness of screw.

Second Law, when the table moves in uniform motion, the Coulomb and viscous frictions of the $y_{1}$ and $y_{2}$ axes are equal to drive forces of motors. The velocities of dual axes are given at various constant velocities within $5 \mathrm{~mm} / \mathrm{s} \sim 14 \mathrm{~m} / \mathrm{min}$ and the frictions can be easily obtained by the feedback drive forces of dual motors. Then, the measurement data can be processed with MATLAB nonlinear fitted function. The identification frictions of dual axis are fitted by practical detection results, as shown in Figure 7. As the velocity increases, friction starts decreasing, and as the velocity exceeds a certain critical value, which is called Stribeck velocity, friction starts to rise gradually again. The fitted parameters of friction model are shown in Table 1.

The equivalent mass of each axis can be estimated by moving the $y_{1}$ and $y_{2}$ at constant acceleration cycles and making the center of gravity of the sliding stage in the geometric center. In this case, the sliding stage is located on the middle of dual axis, and there is no workpiece mounted on the stage, which means $\ddot{y}_{1}=\ddot{y}_{2}, \theta_{g z} \doteq 0$, and $\theta_{g x}=0$.
Then, the variation mass can be derived as

$$
\begin{aligned}
& m_{y 1}=\left(k_{e}\left(y_{1}-y_{s 1}\right)-c_{y_{1}} \operatorname{sign}\left(\dot{y}_{1}\right)\right) \ddot{y}_{1}^{-1}, \\
& m_{y 2}=\left(k_{e}\left(y_{2}-y_{s 2}\right)-c_{y 2} \operatorname{sign}\left(\dot{y}_{2}\right)\right) \ddot{y}_{2}^{-1} .
\end{aligned}
$$

The displacements of $y_{s i}$ and $y_{i}$ can be obtained by the feedback of encoders and grating scales in each axis.

The rotary inertia in the inertia matrix is estimated by the formula of moment of inertia.

$$
\begin{aligned}
& J_{g z}=\frac{\left(m_{y 1}+m_{y 2}+m_{s}\right) L^{2}}{12}, \\
& J_{g x}=\frac{\left(m_{y 1}+m_{y 2}+m_{s}\right) a^{2}}{12} .
\end{aligned}
$$

The identified parameters are given in Table 1.

\section{Test of Proposed Dynamics Model for DDFS DCG}

3.1. Model Parameters. Once the parameters are identified by NI vibration test platform, the modal test and dynamic measurement can be compared and analyzed by simulation and experiments. The experiments are carried out on an industry milling machine based on DCG DDFS. The milling machine is shown in Figure 1. The physical parameters of DCG DDFS are identified in Table 1. The servo parameters of adopted PMSMs are listed in Table 2. The body is subject to mechanical friction with a viscous friction coefficient of $F_{v 1}$ $=0.0072 \mathrm{Ns} / \mathrm{m}$ and a Coulomb friction level of $F_{C 1}=50 \mathrm{~N}$.

To investigate the comparison between simulation and experiment results, different parameters are separated from the influence of natural resonant and feed performance. The height of center of gravity $Z_{q}$ and the position along $x$-axis $X_{g}$ are discussed, respectively. The proposed typical gantry stage with DCG DDFS is illustrated in Figure 8.

3.2. Modal Test and Comparison. To investigate the accuracy of the proposed mechanical-coupled model, the natural frequency is tested by modal experiment. The variation of the acceleration vibration response of the dual-driving DCG feed system is measured by NI vibration measurement 


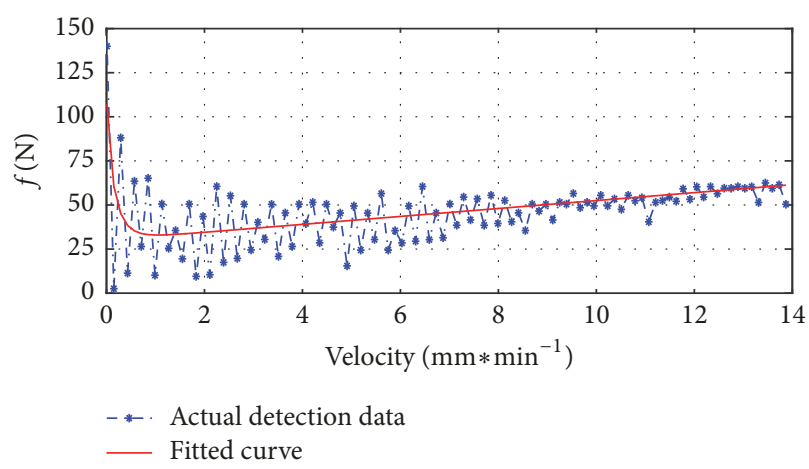

(a)

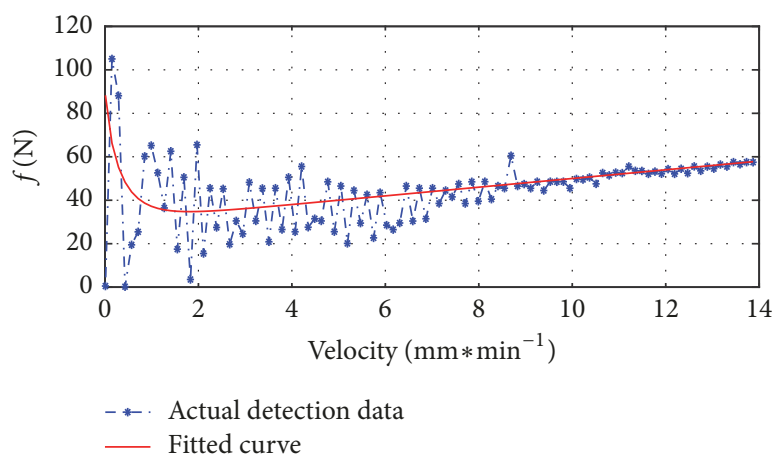

(b)

FIgURE 7: (a) Friction measuring and fitting of axis $y_{1}$; (b) Friction measuring and fitting of axis $y_{2}$.

TABLE 1: Physical parameters of the dual-driving system.

\begin{tabular}{lcc}
\hline Name & Value & Description \\
\hline$m_{y 1}$ & $7.53 \mathrm{~kg}$ & Mass of the axis $y_{1}$ \\
$m_{y 2}$ & $7.61 \mathrm{~kg}$ & Mass of the axis $y_{2}$ \\
$m_{s}$ & $510 \mathrm{~kg}$ & Mass of the sliding stage \\
$J_{g z}$ & $78 \mathrm{~kg} \cdot \mathrm{m}^{2}$ & Rotary inertia of the stage \\
$J_{g x}$ & $51 \mathrm{~kg} \cdot \mathrm{m}^{2}$ & Pitch inertia of the stage \\
$k_{e}$ & $1631.8 \mathrm{~N} \cdot \mathrm{m} / \mathrm{rad}$ & Equivalent stiffness of screw \\
$k_{h z}$ & $1572.5 \mathrm{~N} \cdot \mathrm{m} / \mathrm{rad}$ & Support stiffness of slider \\
$k_{b x}$ & $2.6734 e 6 \mathrm{~N} \cdot \mathrm{m} / \mathrm{rad}$ & Lateral stiffness of slider \\
$L$ & $0.8 \mathrm{~m}$ & Distance of dual screws \\
$a$ & $0.45 \mathrm{~m}$ & Axial distance of the sliders \\
$r_{g}$ & $5 \mathrm{~mm}$ & Screw lead \\
$F_{s 1}$ & $117.7352 \mathrm{~N}$ & Maximum static friction of $y_{1}$ \\
$F_{s 2}$ & $102.1365 \mathrm{~N}$ & Maximum static friction of $y_{2}$ \\
$F_{c 1}$ & $30.2017 \mathrm{~N}$ & Coulomb friction of axis $y_{1}$ \\
$F_{c 2}$ & $26.8520 \mathrm{~N}$ & Coulomb friction of axis $y_{2}$ \\
$F_{v 1}$ & $0.0052 \mathrm{Ns} / \mathrm{m}$ & Viscosity coefficient of axis $y_{1}$ \\
$F_{v 2}$ & $0.0060 \mathrm{Ns} / \mathrm{m}$ & Viscosity coefficient of axis $y_{2}$ \\
$v_{\sigma 1}$ & $73.2533 \mathrm{~mm} / \mathrm{min}$ & Stribeck velocity of axis $y_{1}$ \\
$v_{\sigma 2}$ & $109.3258 \mathrm{~mm} / \mathrm{min}$ & Stribeck velocity of axis $y_{2}$ \\
\hline
\end{tabular}

TABLE 2: Motor parameters.

\begin{tabular}{lccc}
\hline Name & Motor $y_{1}$ & Motor $y_{2}$ & Description \\
\hline$J$ & 0.000167 & 0.000165 & Inertia kg.m $^{2}$ \\
$B$ & 0.00023 & 0.00019 & Damping kg.m ${ }^{2} / \mathrm{sec}$ \\
$k_{t}$ & 1.37 & 1.37 & Torque coefficient NmA \\
$k_{a}$ & 8.4355 & 7.7686 & Amplifier A/V \\
\hline
\end{tabular}

instrument. The hammer is used to simulate with a $3 \mathrm{~Hz}$ test bandwidth. Four three-dimensional acceleration sensors are assembled on the four corners of the sliding stage, so that the torsion and pitch vibration can be measured. The DDFS DCG is shown in Figure 8 and the modal experiment diagram is shown in Figure 9. When the height of center of gravity is zero, the simulation and experiment results of natural frequencies are shown in Tables 3 and 4.
In order to study the influence of height of center of gravity on natural frequencies, the stage is carried on different workpiece and the height of center of gravity is changed from 0 to $300 \mathrm{~mm}$.

The height of center of gravity is a structure parameter defined above. To facilitate the analysis of height of center of gravity, the changing of inertia and jointed parameters is assumed to have no related effect. 
TABLE 3: Natural frequencies and modes of vibration.

\begin{tabular}{lccc}
\hline Eigenvalue & Natural frequency & Vector & Mode of vibration \\
\hline$w_{1}$ & $0 \mathrm{~Hz}$ & {$[1,1,0,0,0]^{T}$} & Axial \\
$w_{2}$ & $48.4 \mathrm{~Hz}$ & {$[1,-1,0,-0.55,0]^{T}$} & Low-order torsion \\
$w_{3}$ & $147.5 \mathrm{~Hz}$ & {$[0.35,0.35,1,-0.05,0]^{T}$} & Axial and torsion \\
$w_{4}$ & $317.2 \mathrm{~Hz}$ & {$[0.03,0.03,0,1,0]^{T}$} & High-order torsion \\
$w_{5}$ & $341.6 \mathrm{~Hz}$ & {$[0,0,0,0.42,1]$} & Pitch and torsion \\
\hline
\end{tabular}

TABLE 4: Comparison of simulation and modal experiment.

\begin{tabular}{lccc}
\hline Mode of vibration & Prediction natural frequency & Experiment natural frequency & Relative error \\
\hline Axial & - & - & - \\
Low-order torsion & $48.4 \mathrm{~Hz}$ & $51.7 \mathrm{~Hz}$ & $6.3 \%$ \\
Axial and torsion & $147.5 \mathrm{~Hz}$ & $136.2 \mathrm{~Hz}$ & $8.3 \%$ \\
Pitch & $317.2 \mathrm{~Hz}$ & $284.4 \mathrm{~Hz}$ & $11.5 \%$ \\
Pitch and torsion & $341.6 \mathrm{~Hz}$ & $302.2 \mathrm{~Hz}$ & $13.0 \%$ \\
\hline
\end{tabular}

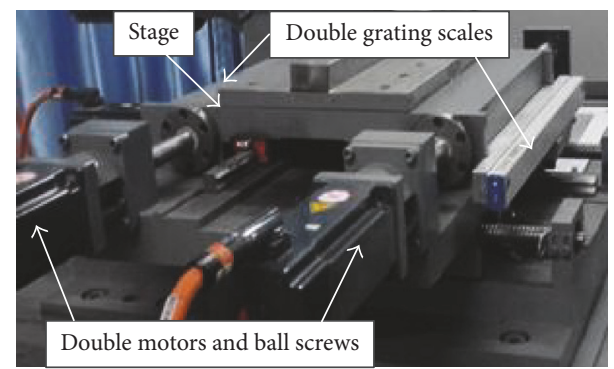

FIgURe 8: Photograph of DDFS DCG.

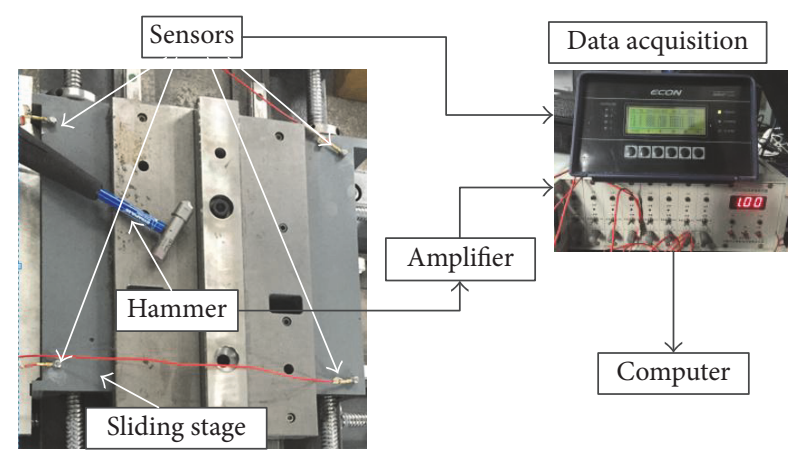

FIgURE 9: Diagram of modal test.

The simulated and experimental results of variation heights of center of gravity effect on each natural frequency are shown in Figure 10. As can be seen in Figure 10, the increase of height of center of gravity has relatively large effect on 3rd (axial and torsion) and 5th (pitch and torsion) vibration. As the height of center of gravity increases, the axial and torsion vibration is reduced from $147.5 \mathrm{~Hz}$ to $123.7 \mathrm{~Hz}$. The pitch and torsion vibration is increased from $341.6 \mathrm{~Hz}$ to $397.9 \mathrm{~Hz}$. In addition, the low-order torsion and highorder torsion vibrations are constant as the height of center of gravity increased.
From Figures 11 and 12, the comparison results show the similarity of the proposed model and actual experiment. The axial and torsion vibration of experiment is reduced from $136.2 \mathrm{~Hz}$ to $112.9 \mathrm{~Hz}$ with the increasing of height of center of gravity. Meanwhile, the experimental pitch and torsion vibration are increased from $302.2 \mathrm{~Hz}$ to $361.7 \mathrm{~Hz}$. The simulation errors confirm that the trend of experiment and the range is also approximate, which illustrates that the model can reflect the actual operation of DDFS.

\section{DCG Dual-Driving Synchronous Feed Experiment}

In this section, the dynamic model-based CCSMC method which considers the synchronous errors is proposed. The synchronous errors and pitch vibrations are measured by laser interferometer and grating scale. The experimental and simulated results are compared to validate the proposed model. Figure 13 is the overall diagram of dynamic modelbased CCSMC.

4.1. Dynamic Model-Based Sliding Mode Control System. For its advantages of completely insensitive to modeling inaccuracies, SMC is particularly suited into nonlinear system. The control objective is to generate a robust sliding mode controller to force the actual motion position to track the given bounded desired reference trajectory and then guarantee that the tracking error $E_{(t)}=Y_{(t)}-Y_{\text {ref }}$ converges asymptotically to zero.

In the practical scenario, the displacements of each axis $Y=\left(y_{1}, y_{2}, x, \theta_{g z}, \theta_{g x}\right)^{T}$ are measurable. In order to assist with the designing of synchronous controller, the redundancy dynamic model can be simplified as

$$
\ddot{Y}=M_{5 \times 5}^{-1}\left(U+F_{5}-K_{5 \times 5} Y\right) \text {, }
$$

where $M_{5 \times 5}^{-1}, K_{5 \times 5}$, and $F_{5}$ are the simplified inertia (14), stiffness (15), and the force (16) matrices. 


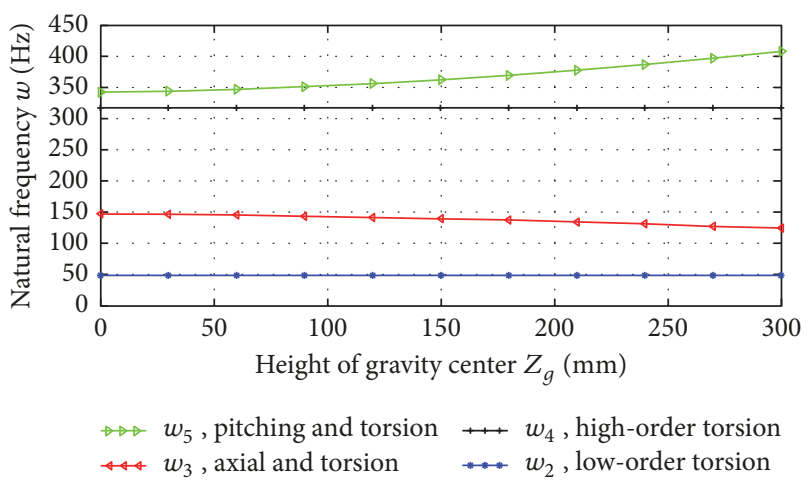

FIGURE 10: Simulation results of height of center of gravity on natural frequency.

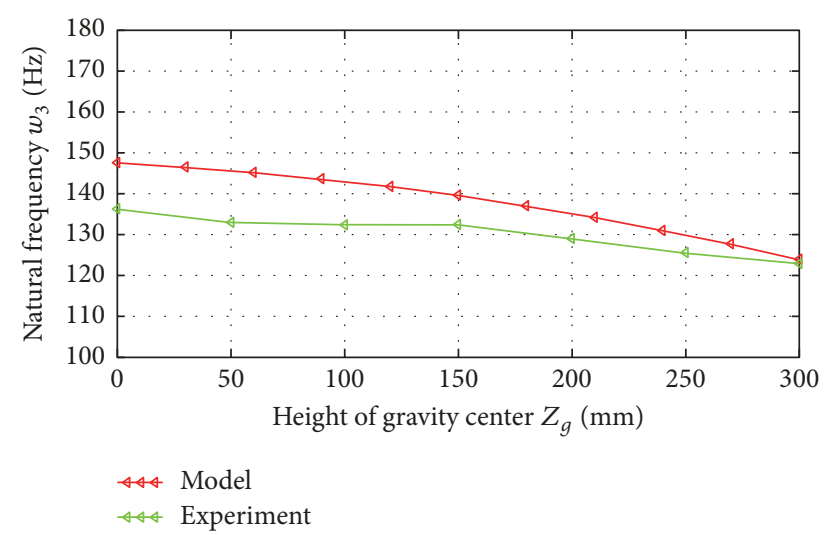

FIGURE 11: Comparison of pitch and torsion vibration.

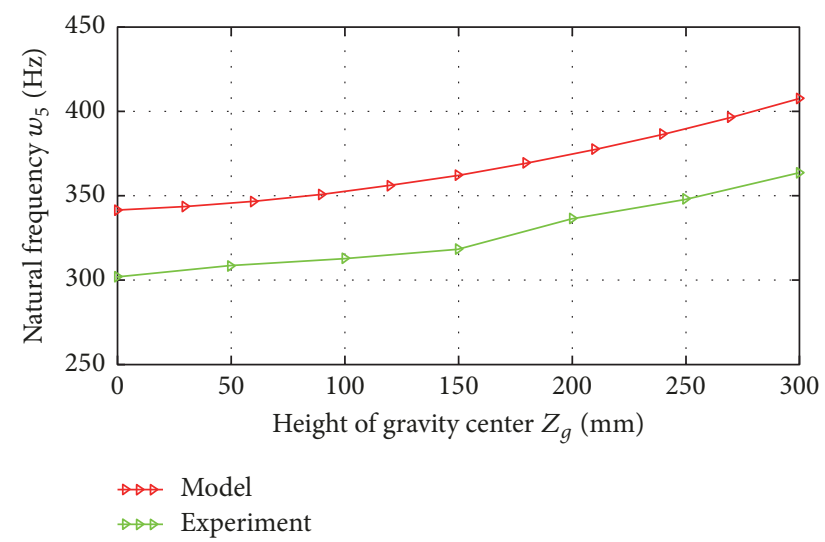

FIGURE 12: Comparison of axial and torsion vibration.

In the DDFS DCG, the control problem is to establish a control law to guarantee the convergence of both the tracking error and synchronous error to zero simultaneously. Hence, the sliding surface is defined as follows:

$$
S_{(t)}=\lambda_{i} E_{(t)}+\dot{E}_{(t)},
$$

where $E_{(t)}=Y_{(t)}-Y_{\text {ref }}$ is defined as the error between actual measurements and reference value; $\lambda_{i}$ is the sliding surface parameter, which should be a positive constant like a transmission gain to determine the convergence speed of the dynamic error on the sliding surface; $i$ depends on the order of $E$.

Taking the derivative of (24) and using (23), the general sliding surface can be obtained.

$$
\begin{aligned}
\dot{S} & =\lambda_{i} \dot{E}_{(t)}+\ddot{E}_{(t)} \\
& =\lambda_{i} \dot{E}_{(t)}+\ddot{Y}_{\text {ref }}-M_{5 \times 5}^{-1}\left(U_{(t)}+F_{5}-K_{5 \times 5} Y\right) .
\end{aligned}
$$

It follows from $\dot{S}_{i(t=0)}=0$ that the equivalent control law can be derived:

$$
U_{\text {eq }}=\lambda_{i} M_{5 \times 5} \dot{E}_{(t)}+M_{5 \times 5} \ddot{Y}_{\text {ref }}-F_{5}+K_{5 \times 5} Y .
$$

A switching control law makes it possible to guarantee the stability of the system, which is defined as

$$
U=U_{\text {eq }}+U_{\text {sc }} \text {, }
$$

where $U_{s c}$ is the switching control function and the traditional SMC adopted signum switching function as switching function, which will improve the robustness and uncertainty disturbances; however, the discontinuous switching can also injects chattering phenomenon into the system, and this chattering introduces undesirable high frequency dynamics in the dual-driving feed system. According to the signum function switch between -1 and +1 , a continuous saturation function is adopted to reduce chattering phenomena with the same function domain.

$$
U_{\mathrm{sc}}=\kappa_{i} \tanh \left(\frac{S}{\varphi_{i}}\right) .
$$

Based on the sliding surface function in the conventional SMC, the switching control law of proposed integral SMC is given as

$$
\begin{aligned}
U_{i}= & \lambda_{i} M_{5 \times 5} \dot{E}_{(t)}+M_{5 \times 5} \ddot{Y}_{\text {ref }}-F_{5}+K_{5 \times 5} Y \\
& +\kappa_{i} \tanh \left(\frac{S}{\varphi_{i}}\right) .
\end{aligned}
$$
(16) in

The Lyapunov function can be expanded by substituting

$$
\dot{V}=S_{i}\left[\lambda_{i} \dot{E}_{(t)}+\ddot{Y}_{\text {ref }}-M_{5 \times 5}^{-1}\left(U_{(t)}+F_{5}-K_{5 \times 5} Y\right)\right] .
$$

To ensure the derivative of Lyapunov function to be negative, the following control equation should be satisfied:

$$
\lambda_{i} \dot{E}_{(t)}+\ddot{Y}_{\text {ref }}
$$

$$
-M_{5 \times 5}^{-1}\left(U_{(t)}+F_{5}-K_{5 \times 5} Y\right) \begin{cases}<0 & \text { for } S>0 \\ =0 & \text { for } S=0 \\ >0 & \text { for } S<0 .\end{cases}
$$

By substituting (29) into (30),

$$
\dot{V}=S_{i} \kappa_{i} \tanh \left(\frac{S_{i}}{\varphi_{i}}\right) .
$$

As $\tanh \left(S_{i} / \varphi_{i}\right)$ ranges from -1 to +1 and is only located in the first and third quadrant. Apparently, the sign of $S_{i}$ and $\tanh \left(S_{i} / \varphi_{i}\right)$ is always sameness, which implies that $\dot{V}$ is negative semidefinite. 


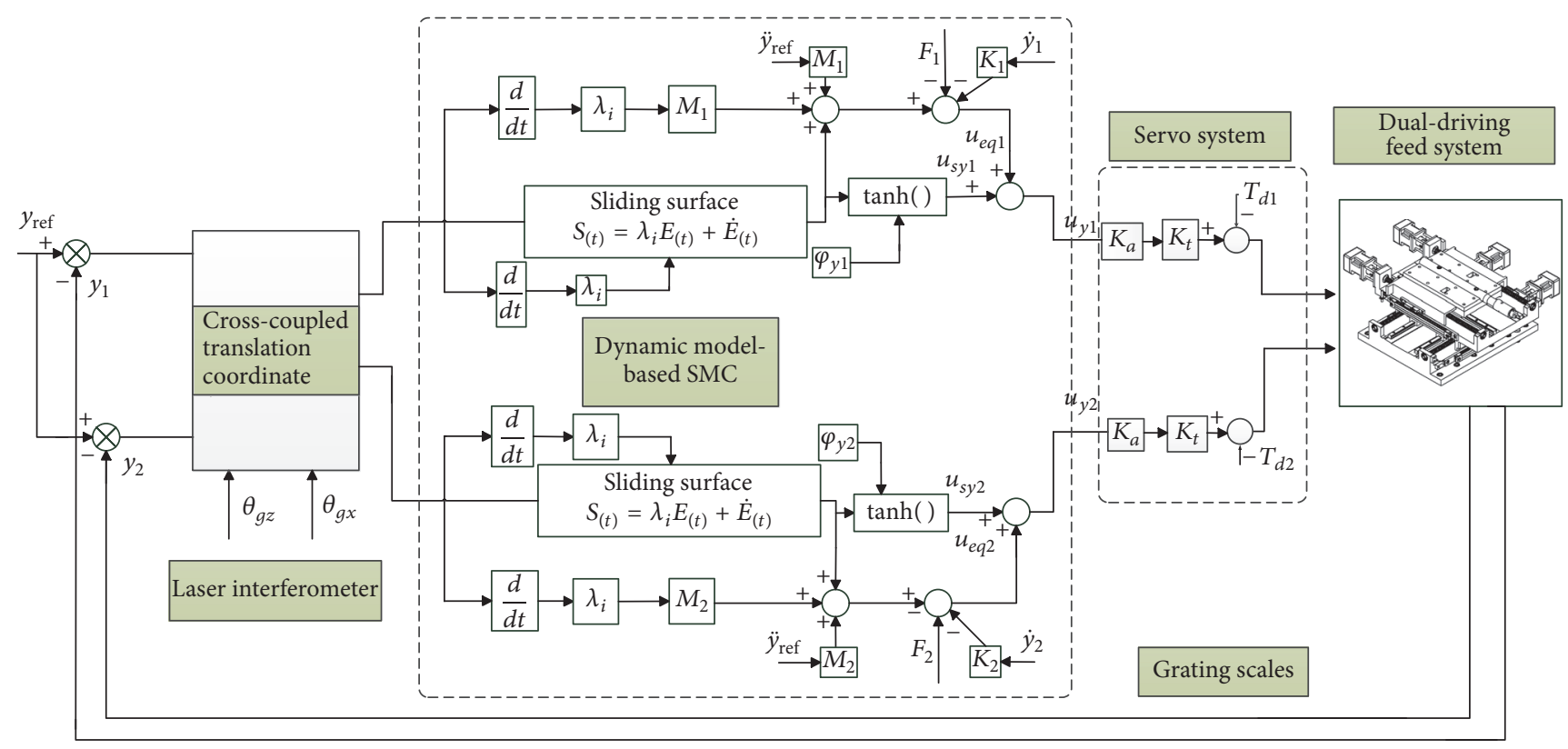

FIgURE 13: Diagram of dynamic model-based CCSMC.

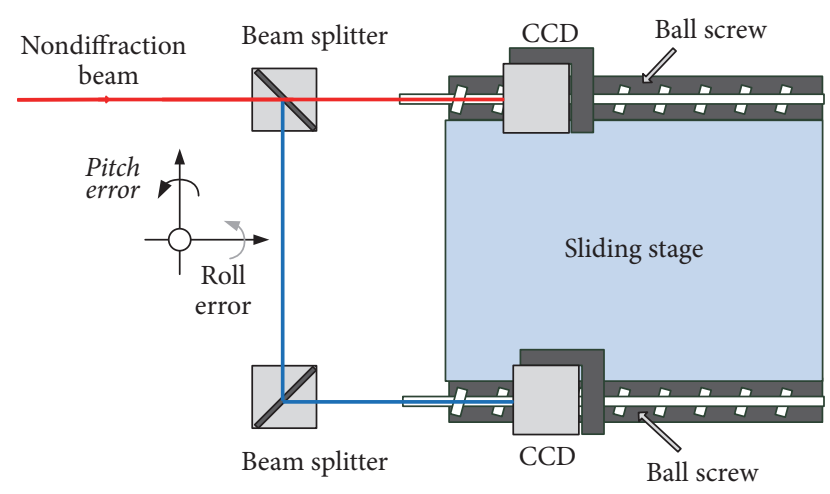

FIGURE 14: Schematic diagram of pitch error measurement.

4.1.1. Pitch Errors Measurement and Analysis. It is known that the dual-driving performance is expressed by the tracking and synchronization errors. However, the pitch vibrations are also crucial to analyze the feed accuracy of DDFS. In particular, when different workpiece is carried on the stage and the center of gravity is changed in height direction, the pitch errors are generated and would intensely reduce the feed precision.

The pitch errors are evaluated by using both the developed dynamic model and laser interferometer. The rotation angle around $z$-axis $\theta_{g z}$ can be deduced by measuring the axial displacements of $y_{1}$ and $y_{2}$. Nevertheless, the pitch error around $x$-axis and roll error around $y$-axis are hardly to be obtained by the normal measuring method which using double grating scales mounted on the both sides of the stage.

A novel pitch angle measurement method of DCG DDFS is proposed in this section. Figures 14 and 15 show the outline and photograph of the measurement scheme. The whole

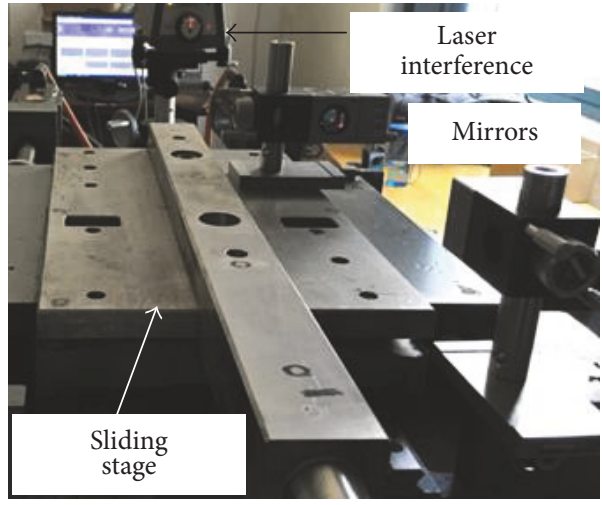

Figure 15: Measurement of laser interference.

measurement system is integrated on the DCG DDFS. In the setup, a laser interferometer is employed as the light source. Each interference mirror is mounted on the fixed table. The reflective mirrors are mounted on the sliding stage to make the measurement axis of the interferometer coincide with the mirrors.

The original pitch error $\theta_{g x}$ of each axis is determined as the assembly accuracy. Although the initial deviations of heights of dual axis $\theta_{g x}^{0}$ are calibrated to be same, the angle errors are inevitably existed. The pitch vibration errors of each axis at any position of the travel can be expressed as

$$
\theta_{g x}=\theta_{g x}^{0}+\Delta \theta_{g x}
$$

The pitch errors are obtained by the simulation and practical measurement. The results are changed from variation located positions of sliding stage along $x$-axis, from the middle position to left-hand (or right-hand side). On the 

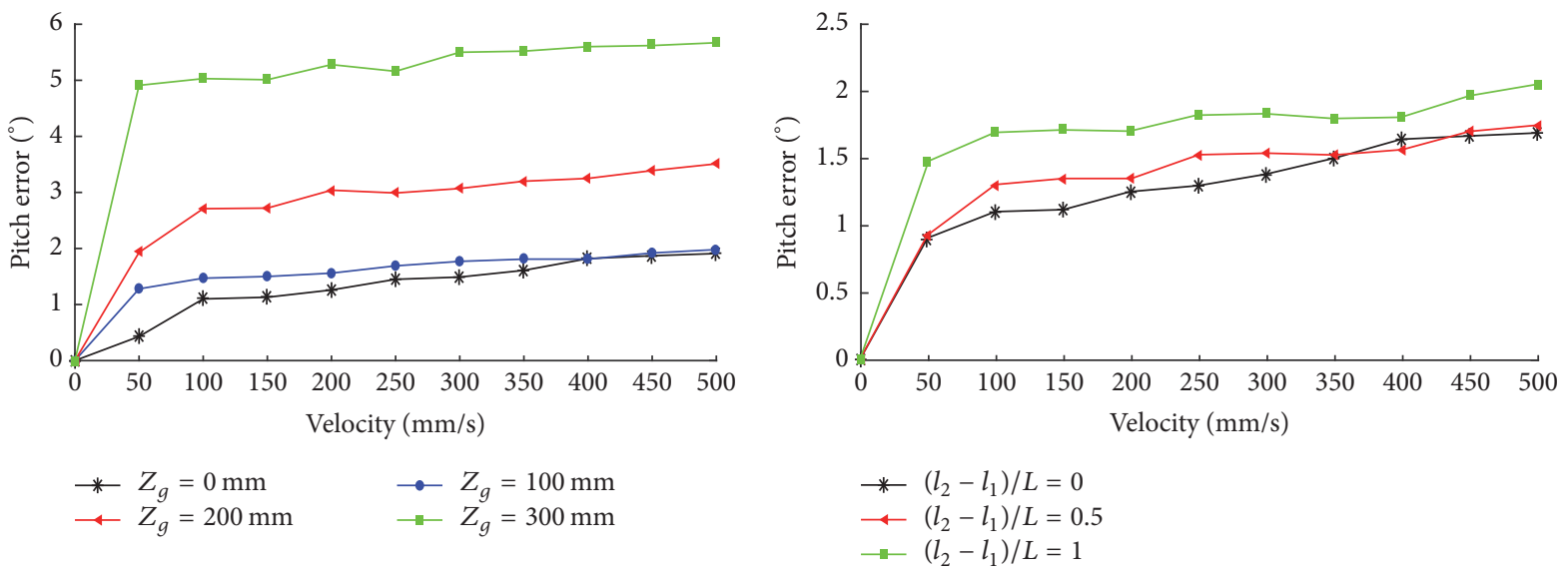

FIGURE 16: Pitch errors change with different position of center of gravity. Feed speed from 0 to $500 \mathrm{~mm} / \mathrm{s}$.

TABLE 5: Comparison between simulation results and practical measurement results.

\begin{tabular}{|c|c|c|c|c|}
\hline $\begin{array}{l}\text { Displacement in } x \text {-axis } \\
\left(l_{2}-l_{1}\right)\end{array}$ & $\begin{array}{l}\text { Height of center } \\
\text { of gravity } Z_{g} \\
(\mathrm{~mm})\end{array}$ & $\begin{array}{l}\text { Maximum } \\
\text { simulation } \\
\text { results } \theta_{g x}\left({ }^{\circ}\right)\end{array}$ & $\begin{array}{c}\text { Maximum } \\
\text { experiment } \\
\text { pitch error } \theta_{g x} \\
\left(^{\circ}\right) \\
\end{array}$ & Relative error \\
\hline 0 & 0 & $1.06 E-3$ & $1.1 E-3$ & $3.6 \%$ \\
\hline $0.25 \mathrm{~L}$ & 0 & $1.29 E-3$ & $1.33 E-3$ & $3.0 \%$ \\
\hline$L$ & 0 & $1.58 E-3$ & $1.69 E-3$ & $6.5 \%$ \\
\hline 0 & 150 & $2.07 E-3$ & $2.2 E-3$ & $5.9 \%$ \\
\hline 0 & 300 & $4.35 E-3$ & $5.1 E-3$ & $14.7 \%$ \\
\hline $0.25 \mathrm{~L}$ & 150 & $2.44 E-3$ & $2.6 E-3$ & $6.2 \%$ \\
\hline $0.25 \mathrm{~L}$ & 300 & $4.67 E-3$ & $5.4 E-3$ & $13.5 \%$ \\
\hline$L$ & 150 & $2.56 E-3$ & $2.7 E-3$ & $5.2 \%$ \\
\hline$L$ & 300 & $5.07 E-3$ & $5.9 E-3$ & $14.1 \%$ \\
\hline $\begin{array}{l}\text { Maximum variation by } \\
\text { displacement in } x \text {-axis }\end{array}$ & - & $0.52 E-3$ & $0.59 E-3$ & $11.9 \%$ \\
\hline $\begin{array}{l}\text { Maximum variation by } \\
\text { height of center of gravity } \\
\text { in } z \text {-axis }\end{array}$ & - & $3.49 E-3$ & $4.21 E-3$ & $17.1 \%$ \\
\hline
\end{tabular}

other hand, the change of height of center of gravity can directly increase the pitch errors. According to the practical processing requirements, the center of gravity is raised from $0 \mathrm{~mm}$ to $300 \mathrm{~mm}$. In order to make specific analysis of the position of center of gravity, the influences of the mass and inertia are not discussed here. The maximum pitch errors are measured at velocity of $100 \mathrm{~mm} / \mathrm{s}$ and acceleration of $1 \mathrm{~m} / \mathrm{s}^{2}$.

The comparison of simulation and practical measurement is shown in Table 5. And the pitch error change with different position of center of gravity is shown in Figure 16. From the results we can notice the following:

(1) The simulated pitch errors are close to the experiment ones, and the relative error is about $3 \%$ to $15 \%$. When the height of center of gravity is constant, the maximum pitch errors of simulation and experiment are increased when the sliding stage is moved from middle position to the left-hand (or right-hand side). When the position of sliding stage along $x$-axis is constant, the pitch errors are increased with the increase of the height of center of gravity.

(2) From Figure 15, the pitch errors are mainly caused by the increase of center of gravity, which is inevitable when different workpieces are mounted on the stage. The pitch errors are generated by the joint forces and can be eliminated by the compensation of position tracking control.

4.1.2. Synchronous Errors Measurement and Analysis. A dualdriving feed experiment is developed based on the computercontrolled dual-driving gantry stage as shown in Figure 17. The Beckhoff control card is the core of the control computer. The control card is integrated in PC, so that the control algorithm, motion control, and other NC tasks can be executed at different levels of CPU, which provide a much better realtime communication property. Each amplifier runs in current control mode which has a circle time $0.1 \mathrm{~ms}$. 


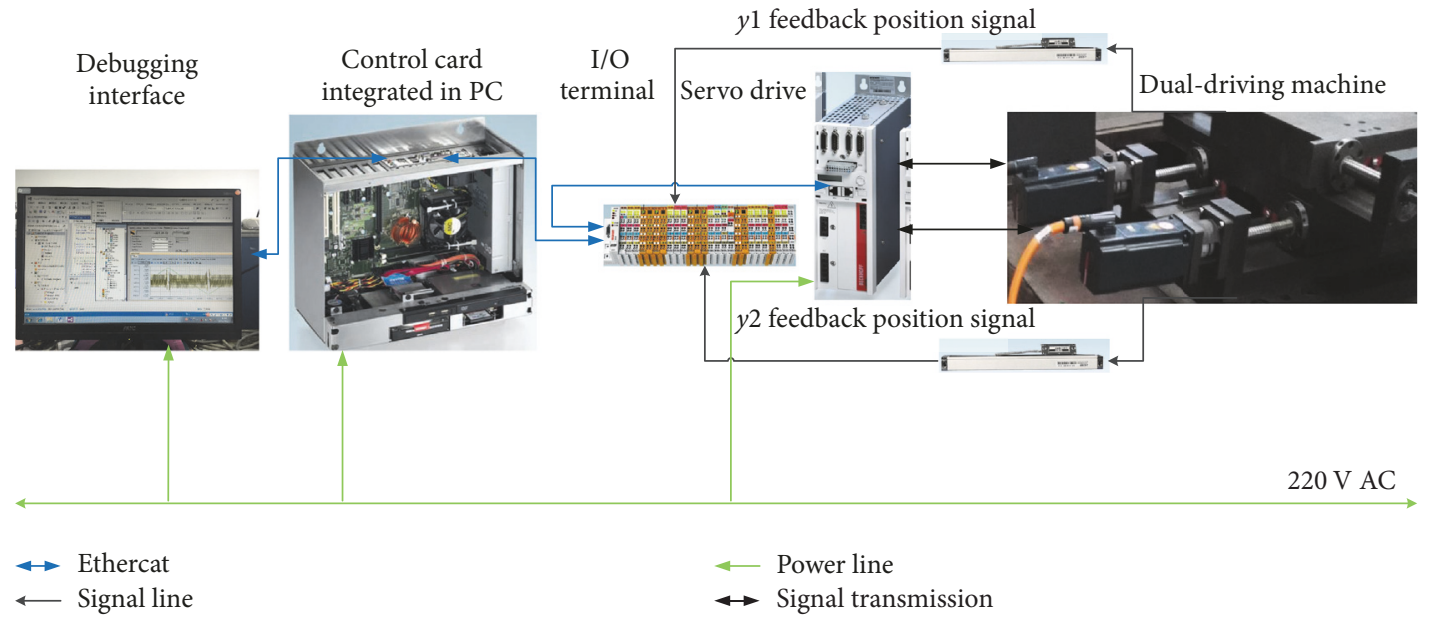

FIGURE 17: Overall hardware architecture.

The feed conditions are conventional where the sliding stage is fed at a speed of $100 \mathrm{~mm} / \mathrm{s}$ by dual axis. Furthermore, the parameters and test conditions are designed to be the same as in the simulation. The resolution of the grating scales is $0.1 \mu \mathrm{m}$ and measuring accuracy is $\pm 1 \mathrm{um}$. After the input command is set, the feedback loop is realized by the encoder sampling at $10[\mathrm{kHz}]$; then the synchronous errors are calculated by the algorithm. Finally, the dual motors are driven by the current commands disposed in the inherent current loop. To describe and clarify the comparison results clearly, the parallel uncoupled control strategy is adopted.

The experimental and simulated synchronous errors of dual axis with an oscillating behavior are studied. While the dual-driving system is feed along $y$-axis, the sliding stage is moved along $x$-axis, and the positions are changed from the middle position $l_{2}=l_{1}$, to left-hand (or right-hand side) $l_{2}-l_{1} \approx L$.

To validate the effectiveness of the proposed model, a normal 2-DOF dynamic model has been developed as (24). In this 2-DOF model, the effects of center of gravity and identified friction are ignored.

$$
\begin{aligned}
D \cdot \ddot{y}+k_{e} y & =F_{1}+F_{2}, \\
F_{1} \cdot \frac{l}{2}-F_{2} \cdot \frac{l}{2}-k_{d} \cdot \theta_{g z} & =J_{g z} \cdot \ddot{\theta}_{g z}, \\
F_{i} & =\frac{2 \pi}{h} T_{M i} .
\end{aligned}
$$

Then the equivalent displacement of dual-driving system can be yielded as

$$
\begin{aligned}
& \left(\begin{array}{l}
\ddot{y}_{1} \\
\ddot{y}_{2}
\end{array}\right)=\left(\frac{1}{L}\left(\begin{array}{cc}
D & 0 \\
0 & J_{t}+\frac{k_{d}}{s^{2}}
\end{array}\right)\left(\begin{array}{cc}
\frac{l}{2} & \frac{l}{2} \\
1 & -1
\end{array}\right)\right)^{-1} \\
& \cdot\left(\begin{array}{cc}
1 & 1 \\
\frac{l}{2} & -\frac{l}{2}
\end{array}\right)\left(\begin{array}{l}
F_{1} \\
F_{2}
\end{array}\right) \text {, }
\end{aligned}
$$

where $D=m_{y 1}+m_{y 2}+m_{s}$ is the total mass; $F_{i}=$ $\left(\begin{array}{l}F_{1} \\ F_{2}\end{array}\right)$ is the drive force, respectively; $k_{d}$ is rotational stiffness of the sliding stage, which points out the rotation error caused by parameters variation and mechanical coupling. The performance tests of the proposed redundancy dynamic model and normal 2-DOF model for the comparison by a dual-driving experiment are shown in Table 6. Comparing the normal 2-DOF model, the relative errors of synchronous error simulated by the proposed model are much reduced. Although the accuracy is slightly increased when the sliding stage is located on the middle, in other two cases, when the sliding stage is located on the $1 / 4$ and one end of the table, the average and max of relative error simulated by the proposed dynamic model are significantly reduced, respectively.

Therefore, the proposed dynamic model can provide significant improvement of the accuracy of dual-driving stage driven by center of gravity.

Then, the practical control performance and synchronization accuracy of proposed dynamic model-based crosscoupled control are testified in the experiment. The proposed control algorithm is applied to the dual-driving servo system by using "C" and MATLAB/SIMULINK language, which will be called by module in the control card. Based on the actual require of manufacturing, two PMSMS are driven by the desired trajectory with $200 \mathrm{~mm}$ peak value, at the speed of $100 \mathrm{~mm} / \mathrm{s}$, as shown in Figure 18. For comparison, the normal cross-coupled control is applied to the DDFS with the same process condition. Figures 19 and 20 depict the experimental results of synchronous control based on the proposed dynamic model-based cross-coupled control and normal cross-coupled control in DDFS DCG.

In comparison to the synchronous performance between proposed dynamic model-based CCSMC and NCCSMC, some phenomena should be noticed in control of dualdriving system:

(1) As can be seen in Figures 19, 20, and 21, the proposed dynamic model-based CCSMC has a favorable tracking response and synchronous performance benefits from the dynamics in handling parameters variation and 
TABLE 6: Performance comparison with variation location in $x$-axis of the sliding stage.

\begin{tabular}{|c|c|c|c|c|c|}
\hline & Experimental results (um) & Proposed DCG model (um) & Relative error & Normal 2-DOF model (um) & Relative error \\
\hline \multicolumn{6}{|c|}{$\left(l_{2}-l_{1}\right) / L=0$} \\
\hline $\operatorname{Max}$ & 9.8 & 9.1 & $7.1 \%$ & 8.9 & $9.2 \%$ \\
\hline Avg & 3.6 & 3.2 & $11.1 \%$ & 3.1 & $13.9 \%$ \\
\hline \multicolumn{6}{|c|}{$\left(l_{2}-l_{1}\right) / L=0.25$} \\
\hline Max & 12.5 & 11.6 & $7.2 \%$ & 9.7 & $22.4 \%$ \\
\hline Avg & 4.1 & 3.7 & $7.3 \%$ & 3.3 & $19.5 \%$ \\
\hline \multicolumn{6}{|c|}{$\left(l_{2}-l_{1}\right) / L=1$} \\
\hline Max & 14.7 & 12.9 & $12.2 \%$ & 10.3 & $29.9 \%$ \\
\hline Avg & 4.3 & 4.0 & $6.9 \%$ & 3.1 & $27.9 \%$ \\
\hline
\end{tabular}

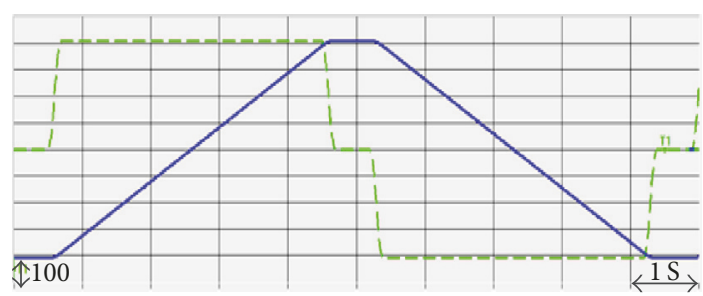

- Trajectory $(\mathrm{mm})$

$---4 * \operatorname{Velocity}(\mathrm{mm} / \mathrm{s})$

(a)

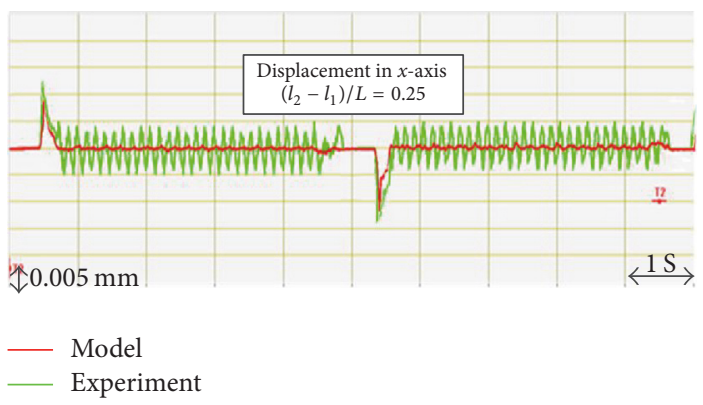

(c)

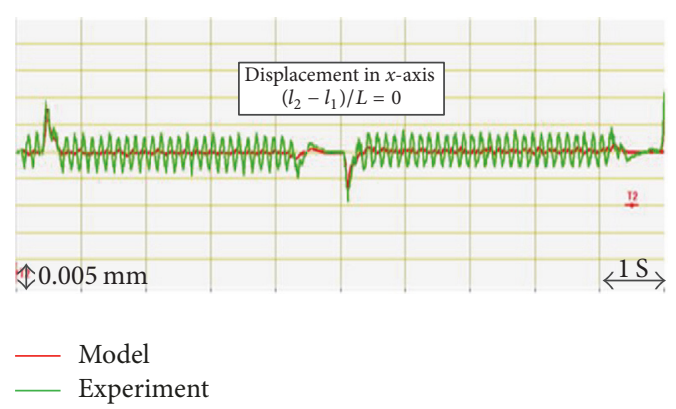

(b)

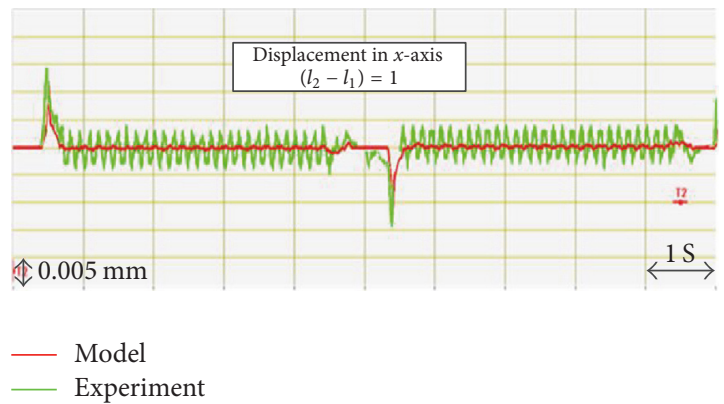

(d)

FIGURE 18: Experiment and simulation results of synchronous errors in dual-driving feed process. (a) Reference trajectory of $y_{1}$ and $y_{2}$; (b) synchronous errors of dual axes when stage is located on $\left(l_{2}-l_{1}\right) / L=0$; (c) synchronous errors of dual axis when stage is located on $\left(l_{2}-l_{1}\right) / L=0.25$; (d) synchronous errors of dual axis when stage is located on $\left(l_{2}-l_{1}\right) / L=1$.

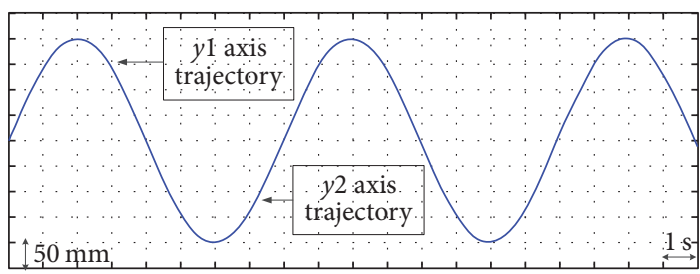

FIGURE 19: Reference trajectory of dual-driving feed experiment.

disturbances. Table 7 shows the details of the comparison results to the NCCSMC, and the proposed dynamic modelbased CCSMC has better performance with respect to the precise synchronization and tracking as well as offering an acceptable performance to the sudden changing of velocity.
(2) The synchronous errors $y_{2}-y_{1}$ are influenced by the position of center of gravity, as shown in Figure 18. The peak value of synchronous errors is increased with the sliding stage moving from the middle position to the left-hand (right-hand side).

\section{Conclusion}

A modeling, identification, and control scheme for dynamic characteristics analysis of DDFS DCG is presented in this paper.

First, a redundancy dynamic model including rotation and pitch errors is developed based on the Lagrange method. The influence of center of gravity is considered in terms of the translation from basic coordinate $\left(x_{0}, y_{0}, z_{0}\right)$ to actual coordinate $\left(x_{g}, y_{g}, z_{g}\right)$. This model accounts for the coupling 
TABLE 7: Performance comparison of dual-driving feed experimental results.

\begin{tabular}{lcr}
\hline Errors/control schemes & & Sinusoidal trajectory \\
Maximum (um) & NCCSMC & Dynamic model-based CCSMC \\
$\quad$ Tracking error & 53.2 & 31.7 \\
$\quad$ Synchronous error & 12.4 & 6.2 \\
Average (um) & & 7.6 \\
$\quad$ Tracking error & 15.1 & 2.1 \\
$\quad$ Synchronous error & 3.8 & \\
\hline
\end{tabular}

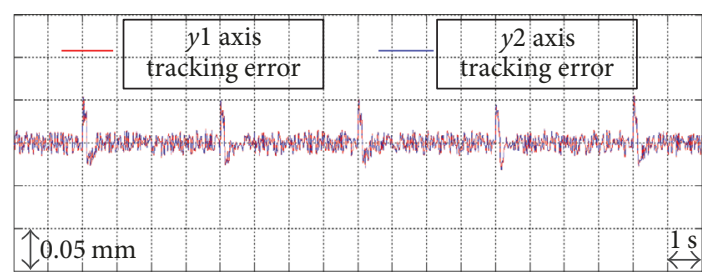

(a)

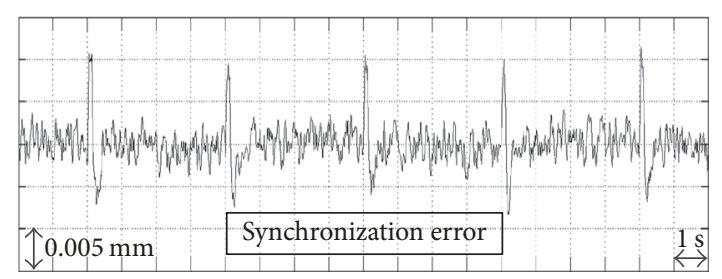

(b)

FIGURE 20: Experimental results of NCCSMC: (a) tracking responses of axis $y_{1}$ and $y_{2}$ and (b) synchronous errors between axis $y_{1}$ and $y_{2}$.

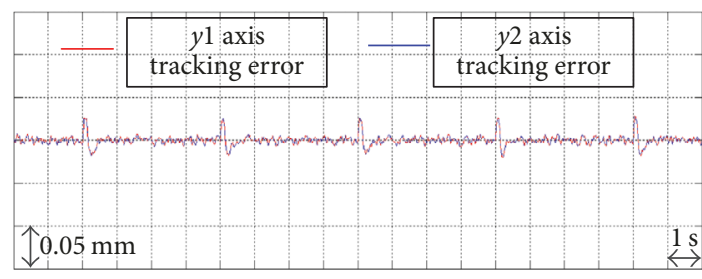

(a)

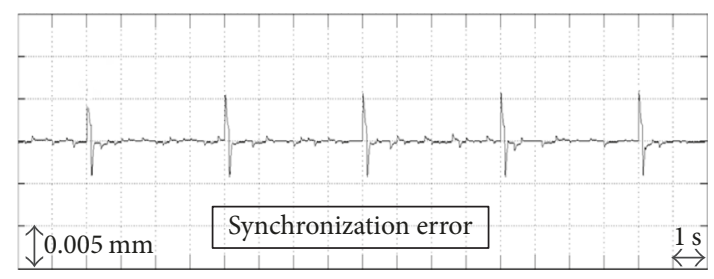

(b)

FIGURE 21: Experimental results of the proposed dynamic model-based CCSMC: (a) tracking responses of axis $y_{1}$ and $y_{2}$ and (b) synchronous errors between axis $y_{1}$ and $y_{2}$.

effects associated with the mechanical coupling between the dual axis and includes the pitch modes caused by joint driving forces. Then the primary parameters (such as inertia and jointed parameters) are identified by the vibration detection system which can be used in the similar DDFS DCG.

The second part of this paper is devoted to the dynamic characteristics test based on a practical DDFS DCG. The relative errors of natural frequencies between simulation and experiment are about $6 \%$ to $13 \%$. With the increase of height of center of gravity, the changing trend of natural frequencies is analyzed. Then, the influence of variation of center of gravity on the dynamic characteristics is discussed with reference to the dual-driving feed experiment. The maximum pitch errors around $x$-axis are obtained by the proposed laser interferometer measurement method. The pitch errors are close between the simulation and the experiment measurement, and the relative error is about $3 \%$ to $15 \%$.

Finally, the dynamic model-based CCSMC is presented for the dual-driving synchronous control. The dynamic model precision is further tested by dual-driving feed experiment. Both the experimental and simulated results show the effectiveness of the proposed model and control scheme.

The main implication of this paper is the dynamic characteristics analysis and control scheme for DDFS DCG. The dynamic model which considers the effect of position of center of gravity has been presented, and the dynamic model-based CCSMC has been developed for DDFS DCG. The predictive natural frequencies and modes can be the reference for filter design and high bandwidth control. Moreover, the dynamic characteristics including pitch errors and synchronous errors are helpful for the tracking and synchronization control and compensation.

\section{Conflicts of Interest}

The authors declare that they have no conflicts of interest.

\section{Acknowledgments}

The work was supported by the National Natural Science Foundation of China (no. 51675393) and National Natural Science Foundation of China (no. 51375197). 


\section{References}

[1] S.-K. Jeong and S.-S. You, "Precise position synchronous control of multi-axis servo system," Mechatronics, vol. 18, no. 3, pp. 129140, 2008.

[2] L. Wang, H. Liu, L. Yang, J. Zhang, W. Zhao, and B. Lu, “The effect of axis coupling on machine tool dynamics determined by tool deviation," The International Journal of Machine Tools and Manufacture, vol. 88, pp. 71-81, 2015.

[3] D. Sepasi, R. Nagamune, and F. Sassani, "Tracking control of flexible ball screw drives with runout effect and mass variation," IEEE Transactions on Industrial Electronics, vol. 59, no. 2, pp. 1248-1256, 2012.

[4] D. A. Vicente, R. L. Hecker, F. J. Villegas, and G. M. Flores, "Modeling and vibration mode analysis of a ball screw drive," The International Journal of Advanced Manufacturing Technology, vol. 58, no. 1-4, pp. 257-265, 2012.

[5] C. S. Teo, K. K. Tan, S. Y. Lim, S. Huang, and E. B. Tay, "Dynamic modeling and adaptive control of a H-type gantry stage," Mechatronics, vol. 17, no. 7, pp. 361-367, 2007.

[6] K. K. Varanasi and S. A. Nayfeh, "The dynamics of leadscrew drives: low-order modeling and experiments," Journal of Dynamic Systems, Measurement, and Control, vol. 126, no. 2, pp. 388-396, 2004.

[7] F.-J. Lin, P.-H. Chou, C.-S. Chen, and Y.-S. Lin, "DSP-based cross-coupled synchronous control for dual linear motors via intelligent complementary sliding mode control," IEEE Transactions on Industrial Electronics, vol. 59, no. 2, pp. 1061-1073, 2012.

[8] D. Sun, "Position synchronization of multiple motion axes with adaptive coupling control," Automatica, vol. 39, no. 6, pp. 9971005, 2003.

[9] H.-K. Park, S.-S. Kim, J.-M. Park, D. Hong, and T.-Y. Cho, "Design of a dual-drive mechanism for precision gantry," KSME International Journal, vol. 16, no. 12, pp. 1664-1672, 2002.

[10] M. D. Kim, K.-H. Lee, K.-T. Nam, and S.-M. Lee, "Design and control of a single-stage dual-actuator system for high-precision manufacturing," Microsystem Technologies, vol. 20, no. 2, pp. 175-183, 2014.

[11] H. Shi, D. Zhang, J. Yang, C. Ma, X. Mei, and G. Gong, "Experiment-based thermal error modeling method for dual ball screw feed system of precision machine tool," The International Journal of Advanced Manufacturing Technology, vol. 82, no. 9-12, pp. 1693-1705, 2016.

[12] F.-J. Lin, P.-H. Chou, C.-S. Chen, and Y.-S. Lin, “Three-degreeof-freedom dynamic model-based intelligent nonsingular terminal sliding mode control for a gantry position stage," IEEE Transactions on Fuzzy Systems, vol. 20, no. 5, pp. 971-985, 2012.

[13] N. Tounsi, T. Bailey, and M. A. Elbestawi, "Identification of acceleration deceleration profile of feed drive systems in CNC machines," The International Journal of Machine Tools and Manufacture, vol. 43, no. 5, pp. 441-451, 2003.

[14] B. Armstrong-Hélouvry, P. Dupont, and C. C. de Wit, "A survey of models, analysis tools and compensation methods for the control of machines with friction," Automatica, vol. 30, no. 7, pp. 1083-1138, 1994.

[15] I. García-Herreros, X. Kestelyn, J. Gomand, R. Coleman, and P.-J. Barre, "Model-based decoupling control method for dualdrive gantry stages: A case study with experimental validations," Control Engineering Practice, vol. 21, no. 3, pp. 298-307, 2013.

[16] M.-F. Hsieh, W.-S. Yao, and C.-R. Chiang, "Modeling and synchronous control of a single-axis stage driven by dual mechanically-coupled parallel ball screws," The International Journal of Advanced Manufacturing Technology, vol. 34, no. 910, pp. 933-943, 2007.

[17] C. Okwudire and Y. Altintas, "Minimum tracking error control of flexible ball screw drives using a discrete-time sliding mode controller," Journal of Dynamic Systems, Measurement, and Control, vol. 131, no. 5, pp. 1-12, 2009.

[18] A. Kamalzadeh and K. Erkorkmaz, "Accurate tracking controller design for high-speed drives," The International Journal of Machine Tools and Manufacture, vol. 47, no. 9, pp. 1393-1400, 2007.

[19] G. Pritschow and N. Croon, "Ball screw drives with enhanced bandwidth by modification of the axial bearing," CIRP Annals Manufacturing Technology, vol. 62, no. 1, pp. 383-386, 2013.

[20] A. Kamalzadeh and K. Erkorkmaz, "Compensation of axial vibrations in ball screw drives," CIRP Annals - Manufacturing Technology, vol. 56, no. 1, pp. 373-378, 2007.

[21] M. Tavan, A. Khaki-Sedigh, M.-R. Arvan, and A.-R. Vali, "Immersion and invariance adaptive velocity observer for a class of Euler-Lagrange mechanical systems," Nonlinear Dynamics, vol. 85, no. 1, pp. 425-437, 2016.

[22] C. E. Okwudire and Y. Altintas, "Hybrid modeling of ball screw drives with coupled axial, torsional, and lateral dynamics," Journal of Mechanical Design, vol. 131, no. 7, pp. 07100210710029, 2009.

[23] T. S. Giam, K. K. Tan, and S. Huang, "Precision coordinated control of multi-axis gantry stages," ISA Transactions ${ }^{\circledR}$, vol. 46, no. 3, pp. 399-409, 2007.

[24] B. Sencer, T. Mori, and E. Shamoto, "Design and application of a sliding mode controller for accurate motion synchronization of dual servo systems," Control Engineering Practice, vol. 21, no. 11, pp. 1519-1530, 2013. 


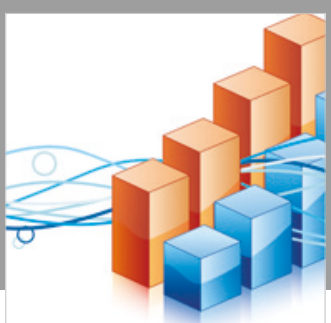

Advances in

Operations Research

\section{-n-m}
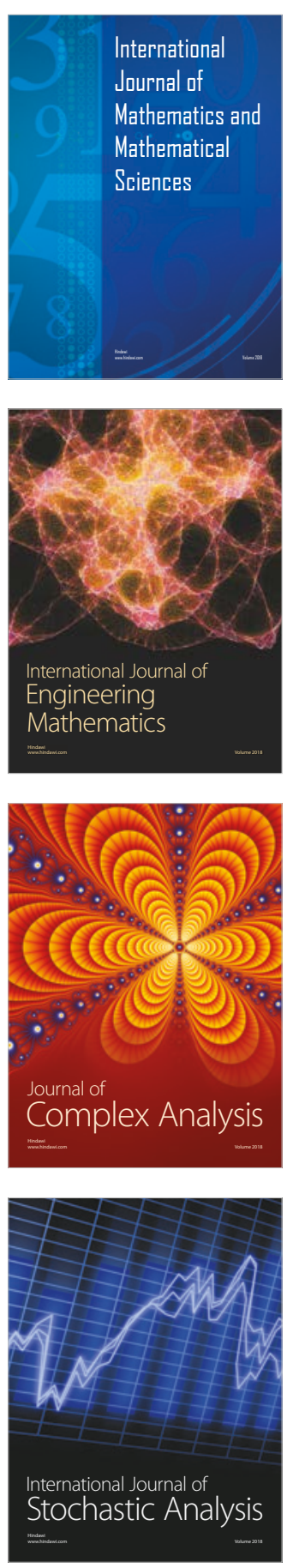
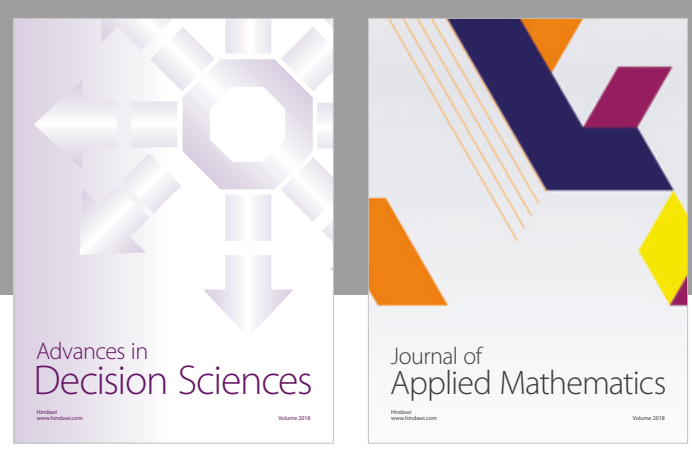

Journal of

Applied Mathematics
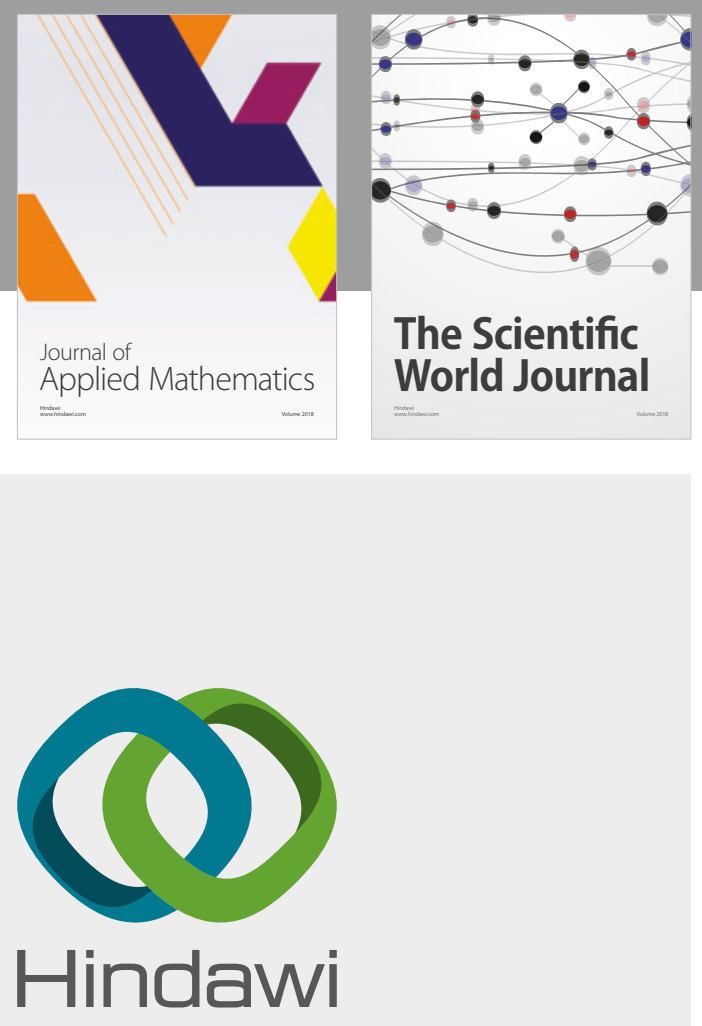

Submit your manuscripts at

www.hindawi.com

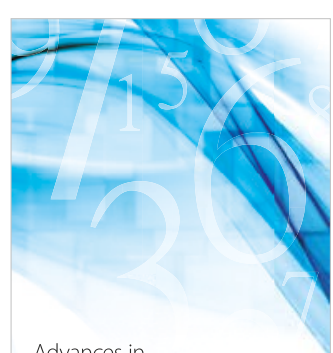

Advances in
Numerical Analysis
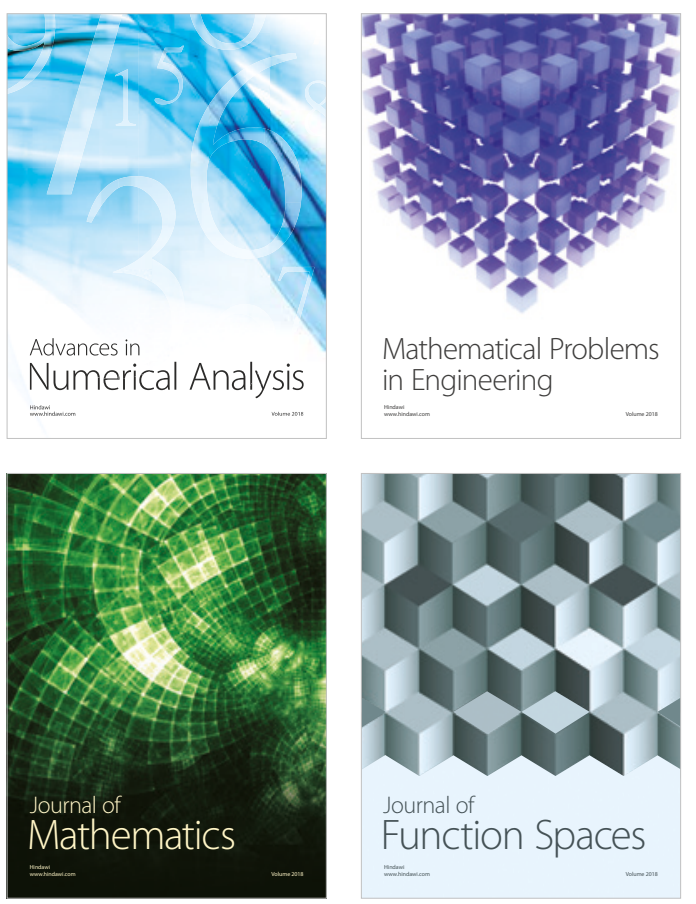

Mathematical Problems in Engineering

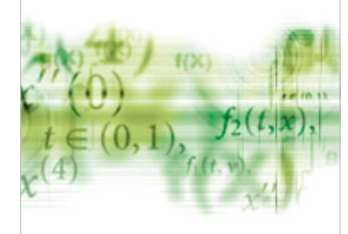

International Journal of

Differential Equations

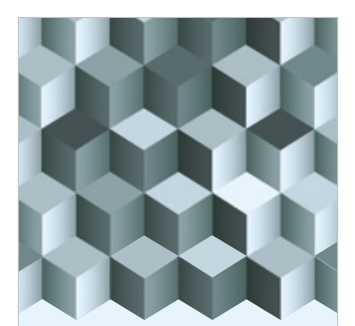

Journal of

Function Spaces

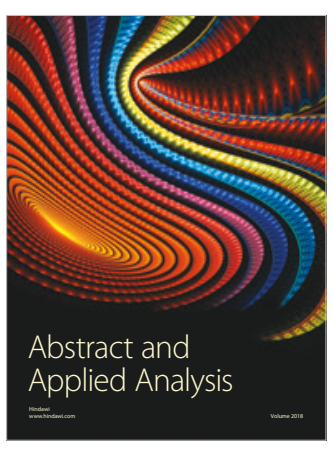

The Scientific

World Journal

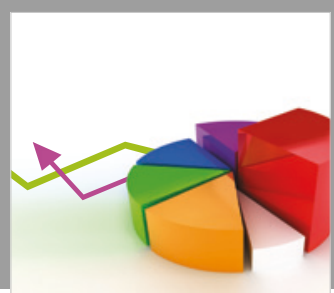

Journal of

Probability and Statistics
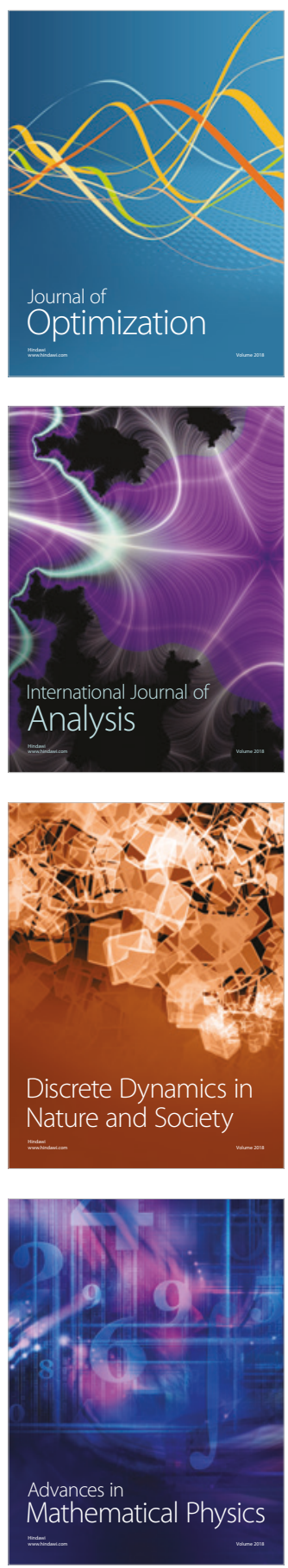